\title{
Symbolic Summation with Single-Nested Sum Extensions
}

\author{
Carsten Schneider* \\ Research Institute for Symbolic Computation \\ J. Kepler University Linz \\ Altenberger Str. 69 \\ A-4040 Linz, Austria \\ Carsten.Schneider@risc.uni-linz.ac.at
}

\begin{abstract}
We present a streamlined and refined version of Karr's summation algorithm. Karr's original approach constructively decides the telescoping problem in $\Pi \Sigma$-fields, a very general class of difference fields that can describe rational terms of arbitrarily nested indefinite sums and products. More generally, our new algorithm can decide constructively if there exists a so called single-nested $\Pi \Sigma$-extension over a given $\Pi \Sigma$-field in which the telescoping problem for $f$ can be solved in terms that are not more nested than $f$ itself. This allows to eliminate an indefinite sum over $f$ by expressing it in terms of additional sums that are not more nested than $f$. Moreover, our refined algorithm contributes to definite summation: it can decide constructively if the creative telescoping problem for a fixed order can be solved in singlenested $\Sigma^{*}$-extensions that are less nested than the definite sum itself.
\end{abstract}

\section{Categories and Subject Descriptors}

I.1.1 [Expressions and Their Simplification]: Simplification of Expressions; I.1.2 [Symbolic and Algebraic Manipulation]: Algebraic algorithms

\section{General Terms}

Algorithms

\section{Keywords}

Difference field extensions, telescoping, creative telescoping

\section{INTRODUCTION}

Let $(\mathbb{F}, \sigma)$ be a difference field, i.e., a field ${ }^{1} \mathbb{F}$ together with a field automorphism $\sigma: \mathbb{F} \rightarrow \mathbb{F}$, and let $\mathbb{K}$ be its constant

*Supported by the Austrian Academy of Sciences, the SFB-grant F1305 of the Austrian FWF, and the FWFForschungsprojekt P16613-N12.

${ }^{1}$ Throughout this paper all fields will have characteristic 0 .

Permission to make digital or hard copies of all or part of this work for personal or classroom use is granted without fee provided that copies are not made or distributed for profit or commercial advantage and that copies bear this notice and the full citation on the first page. To copy otherwise, to republish, to post on servers or to redistribute to lists, requires prior specific permission and/or a fee.

ISSAC'04, July 4-7, 2004, Santander, Spain.

Copyright 2004 ACM 1-58113-827-X/04/0007 ...\$5.00. field, i.e., $\mathbb{K}=$ const $_{\sigma} \mathbb{F}:=\{k \in \mathbb{F} \mid \sigma(k)=k\}$. Then Problem $P F L D E$ plays an important role in symbolic summation.

Problem PFLDE: Solving Parameterized First Order Linear Difference Equations.

Given $a_{1}, a_{2} \in \mathbb{F}^{*}$ and $\left(f_{1}, \ldots, f_{n}\right) \in \mathbb{F}^{n}$. Find all $g \in \mathbb{F}$ and $\left(c_{1}, \ldots, c_{n}\right) \in \mathbb{K}^{n}$ with $a_{1} \sigma(g)+a_{2} g=\sum_{i=1}^{n} c_{i} f_{i}$.

For instance, if one takes the field of rational functions $\mathbb{F}=\mathbb{K}(k)$ with the shift $\sigma(k)=k+1$ and specializes to $n=1, a_{1}=1$ and $a_{2}=-1$, one considers the telescoping problem for a rational function $f_{1}=f^{\prime}(k) \in \mathbb{K}(k)$. Moreover, if $\mathbb{K}=\mathbb{K}^{\prime}(m)$ and $f_{i}=f^{\prime}(m+i-1, k) \in \mathbb{K}^{\prime}(m)(k)$ for $1 \leq i \leq n$, one formulates the creative telescoping problem [15] of order $n-1$ for definite rational sums.

More generally, $\Pi \Sigma$-fields, introduced in [6, 7], are difference fields $(\mathbb{F}, \sigma)$ with constant field $\mathbb{K}$ where $\mathbb{F}:=\mathbb{K}\left(t_{1}\right) \ldots,\left(t_{e}\right)$ is a rational function field and the application of $\sigma$ on the $t_{i}$ 's is recursively defined over $1 \leq i \leq e$ with $\sigma\left(t_{i}\right)=\alpha_{i} t_{i}+\beta_{i}$ for $\alpha_{i}, \beta_{i} \in \mathbb{K}\left(t_{1}\right) \ldots\left(t_{i-1}\right)$; we omitted some technical conditions given in Section 2. Note that $\Pi \Sigma$-fields enable one to describe a huge class of sequences, like hypergeometric terms, as shown in [13], or most d'Alembertian solutions [1, 9], a subclass of Liouvillian solutions [5] of linear recurrences. More generally, $\Pi \Sigma$-fields allow to describe rational terms consisting of arbitrarily nested indefinite sums and products. We want to emphasize that the nested depth of these sums and products gives a measure of the complexity of expressions. This can be carried over to $\Pi \Sigma$-fields by introducing the depth of $t_{i}$ as the number of recursive definition steps that are needed to describe the application of $\sigma$ on $t_{i}$; for more details see Section 2. Moreover, the depth of $f \in \mathbb{F}$ is the maximum depth of the $t_{i}$ 's that occur in $f$, and the depth of $(\mathbb{F}, \sigma)$ is the maximum depth of all the $t_{i}$.

The main result in [6] is an algorithm that solves Problem PFLDE and therefore the telescoping and creative telescoping problem for a given $\Pi \Sigma$-field $(\mathbb{F}, \sigma)$ where the constant field $\mathbb{K}$ is $\sigma$-computable. This means that (1) for any $k \in \mathbb{K}$ one can decide if $k \in \mathbb{Z}$, (2) polynomials in $\mathbb{K}\left[t_{1}, \ldots, t_{n}\right]$ can be factored over $\mathbb{K}$, and (3) one knows how to compute a basis of $\left\{\left(n_{1}, \ldots, n_{k}\right) \in \mathbb{Z}^{k} \mid c_{1}^{n_{1}} \ldots c_{k}^{n_{k}}=1\right\}$ for $\left(c_{1}, \ldots, c_{k}\right) \in \mathbb{K}^{k}$ which is a submodule of $\mathbb{Z}^{k}$ over $\mathbb{Z}$. For instance, any rational function field $\mathbb{K}=\mathbb{A}\left(x_{1}, \ldots, x_{r}\right)$ over an algebraic number field $\mathbb{A}$ is $\sigma$-computable; see [13]. In this paper we will present a streamlined and simplified version of Karr's original algorithm [6] for Problem PFLDE using Bronstein's denominator bound [2] and results from [6, $12,10,11]$. Afterwards we will extend this approach to an algorithm that can solve 
Problem RS: Refined Summation. Given a $\Pi \Sigma$-field $(\mathbb{F}, \sigma)$ with depth $d$, constant field $\mathbb{K}$ and $\left(f_{1}, \ldots, f_{n}\right) \in \mathbb{F}^{n}$. Decide constructively if there are $(0, \ldots, 0) \neq\left(c_{1}, \ldots, c_{n}\right) \in \mathbb{K}^{n}$ and $g \in \mathbb{F}\left(x_{1}\right) \ldots\left(x_{e}\right)$ for $\sigma(g)-g=\sum_{i=1}^{n} c_{i} f_{i}$ in an extended $\Pi \Sigma$-field $\left(\mathbb{F}\left(x_{1}\right) \ldots\left(x_{e}\right), \sigma\right)$ with depth $d$ and $\sigma\left(x_{i}\right)=$ $\alpha_{i} x_{i}+\beta_{i}$ where $\alpha_{i}, \beta_{i} \in \mathbb{F}$.

Suppose we fail to find a solution $g$ with $\sigma(g)-g=f$ in a given $\Pi \Sigma$-field $(\mathbb{F}, \sigma)$ with depth $d$ and $f \in \mathbb{F}^{*}$ with depth $d$, but there exists such an extended $\Pi \Sigma$-field $\left(\mathbb{F}\left(x_{1}\right) \ldots\left(x_{e}\right), \sigma\right)$ and a solution $g$ with depth $d$ for $\sigma(g)-g=f$. Then our new algorithm can compute such an extension with such a solution $g$. As a side result we will show that it suffices to restrict to the sum case, i.e., $\sigma\left(x_{i}\right)-x_{i} \in \mathbb{F}$. In some sense our results shed new constructive light on Karr's Fundamental Theorem [6].

For instance, in Karr's approach [6] one can find the right hand side in (1) only by setting up manually the corresponding $\Pi \Sigma$-field in terms of the harmonic numbers $H_{n}:=$ $\sum_{i=1}^{n} \frac{1}{i}$ and the generalized versions $H_{n}^{(r)}:=\sum_{i=1}^{n} \frac{1}{i^{r}}, r>1$, whereas with our new algorithm the underlying $\Pi \sum$-field is constructed completely automatically. Additional examples are

$$
\begin{gathered}
\sum_{k=1}^{n} \frac{1}{k} \sum_{j=1}^{k} \frac{1}{j} \sum_{i=1}^{j} \frac{1}{i}=\frac{1}{6}\left[H_{n}^{3}+3 H_{n} H_{n}^{(2)}+2 H_{n}^{(3)}\right], \\
\sum_{k=1}^{n} \frac{1}{k} \sum_{i=1}^{k} \frac{1}{H_{i}}=-n+H_{n} \sum_{i=1}^{n} \frac{1}{H_{i}}+\sum_{i=1}^{n} \frac{1}{i H_{i}}, \\
\sum_{k=0}^{a}\left(\sum_{i=0}^{k}\left(\begin{array}{c}
n \\
i
\end{array}\right)\right)^{2}=(n-a)\left(\begin{array}{c}
n \\
a
\end{array}\right) \sum_{i=0}^{a}\left(\begin{array}{c}
n \\
i
\end{array}\right) \\
-\frac{n-2 a-2}{2}\left(\sum_{i=0}^{a}\left(\begin{array}{c}
n \\
i
\end{array}\right)\right)^{2}-\frac{n}{2} \sum_{i=1}^{n}\left(\begin{array}{c}
n \\
i
\end{array}\right)^{2} .
\end{gathered}
$$

Our new approach also refines creative telescoping: we might find a recurrence of smaller order by introducing additional sums with depths smaller than the definite sum.

All these algorithms have been implemented in form of the summation package Sigma in the computer algebra system Mathematica. The wide applicability of this new approach is illustrated for instance in $[9,8,4]$.

\section{REFINED SUMMATION IN $\Pi \Sigma$-FIELDS}

First we introduce some notations and definitions. Let $(\mathbb{F}, \sigma)$ be a difference field with constant field $\mathbb{K}=$ const $_{\sigma} \mathbb{F}$, $\boldsymbol{a}=\left(a_{1}, a_{2}\right) \in \mathbb{F}^{2}$ and $\boldsymbol{f}=\left(f_{1}, \ldots, f_{n}\right) \in \mathbb{F}^{n}$. For any $\boldsymbol{h}=\left(h_{1}, \ldots, h_{n}\right) \in \mathbb{F}^{n}$ and $p \in \mathbb{F}$ we write $\boldsymbol{f} \boldsymbol{h}:=\sum_{i=1}^{n} f_{i} h_{i}$, $\sigma(\boldsymbol{h}):=\left(\sigma\left(h_{1}\right), \ldots, \sigma\left(h_{n}\right)\right)$, and $\boldsymbol{h} p:=\left(h_{1} p, \ldots, h_{n} p\right)$. We call $\boldsymbol{a}$ homogeneous over $\mathbb{F}$ if $a_{1} a_{2} \neq 0$ and $a_{1} \sigma(g)+a_{2} g=0$ for some $g \in \mathbb{F}^{*}$.

Now let $\mathbb{V}$ be a subspace of $\mathbb{F}$ over $\mathbb{K}$ and suppose that $\boldsymbol{a} \neq \mathbf{0}$. Then we define the solution space $\mathrm{V}(\boldsymbol{a}, \boldsymbol{f}, \mathbb{V})$ as the subspace $\left\{\left(c_{1}, \ldots, c_{n}, g\right) \in \mathbb{K}^{n} \times \mathbb{V} \mid a_{1} \sigma(g)+a_{2} g=\sum_{i=1}^{n} c_{i} f_{i}\right\}$ of the vector space $\mathbb{K}^{n} \times \mathbb{F}$ over $\mathbb{K}$. By difference field theory [3], the dimension is at most $n+1$; see also [9, 10]. Therefore Problem PFLDE is equivalent to find a basis of $\mathrm{V}(\boldsymbol{a}, \boldsymbol{f}, \mathbb{F})$. A difference field $\left(\mathbb{E}, \sigma^{\prime}\right)$ is a difference field extension of $(\mathbb{F}, \sigma)$ if $\mathbb{F}$ is a subfield of $\mathbb{E}$ and $\sigma^{\prime}(g)=\sigma(g)$ for $g \in \mathbb{F}$; note that from now $\sigma$ and $\sigma^{\prime}$ are not distinguished anymore.
A difference field extension $(\mathbb{F}(t), \sigma)$ of $(\mathbb{F}, \sigma)$ is a $\Pi$ - (resp. $\left.\Sigma^{*}-\right)$ extension if $\mathbb{F}(t)$ is a rational function field, $\sigma(t)=a t$ $(\sigma(t)=t+a$ resp. $)$ for some $a \in \mathbb{F}^{*}$ and const $\sigma \mathbb{F}(t)=$ const $_{\sigma} \mathbb{F}$. A difference field extension $(\mathbb{F}(t), \sigma)$ of $(\mathbb{F}, \sigma)$ is a $\Sigma$-extension if $\mathbb{F}(t)$ is a rational function field, $\sigma(t)=\alpha t+\beta$ for some $\alpha, \beta \in \mathbb{F}^{*}$, const $_{\sigma} \mathbb{F}(t)=$ const $_{\sigma} \mathbb{F}$, and the following two properties hold: (1) there does not exist a $g \in \mathbb{F}(t) \backslash \mathbb{F}$ with $\frac{\sigma(g)}{g} \in \mathbb{F}$, and (2) if there is a $g \in \mathbb{F}^{*}$ and $n \neq 0$ with $\sigma(g) / g=\alpha^{n}$ then there is also a $g \in \mathbb{F}^{*}$ with $\sigma(g) / g=\alpha$. Note that any $\Sigma^{*}$-extension is also a $\Sigma$-extension; for more details see $[6,7,2,9,13]$. A $\Pi \Sigma$-extension is either a $\Pi$ - or a $\Sigma$-extension.

A difference field extension $\left(\mathbb{F}\left(t_{1}\right) \ldots\left(t_{e}\right), \sigma\right)$ of $(\mathbb{F}, \sigma)$ is a (nested) $\Sigma^{*} / \Pi \Sigma$-extension if $\left(\mathbb{F}\left(t_{1}\right) \ldots\left(t_{i}\right), \sigma\right)$ is a $\Sigma^{*} / \Pi \Sigma$ extension of $\left(\mathbb{F}\left(t_{1}\right) \ldots\left(t_{i-1}\right), \sigma\right)$ for all $1 \leq i \leq e$; for $i=0$ we define $\mathbb{F}\left(t_{1}\right) \ldots\left(t_{i-1}\right)=\mathbb{F}$. Note that $e=0$ gives the trivial extension.

For $\mathbb{H} \subseteq \mathbb{F}$, a $\Pi \Sigma$-extension $\left(\mathbb{F}\left(t_{1}\right) \ldots\left(t_{e}\right), \sigma\right)$ of $(\mathbb{F}, \sigma)$ is single-nested over $\mathbb{H}$, or in short over $\mathbb{H}$, if $\sigma\left(t_{i}\right)=\alpha_{i} t_{i}+\beta_{i}$ with $\alpha_{i}, \beta_{i} \in \mathbb{H}$ for all $1 \leq i \leq e$. A $\Pi \Sigma$-extension of $(\mathbb{F}, \sigma)$ is called single-nested, if it is single-nested over $\mathbb{F}$.

Finally, a $\Pi \Sigma$-field $(\mathbb{F}, \sigma)$ over $\mathbb{K}$ is a $\Pi \Sigma$-extension of $(\mathbb{K}, \sigma)$ with const ${ }_{\sigma} \mathbb{K}=\mathbb{K}$, i.e., const $_{\sigma} \mathbb{F}=\mathbb{K}$.

In [6] alternative definitions of $\Pi \Sigma$-extensions are introduced that allow to decide constructively if an extension $(\mathbb{F}(t), \sigma)$ of $(\mathbb{F}, \sigma)$ is a $\Pi \Sigma$-extension under the assumption that $(\mathbb{F}, \sigma)$ is a $\Pi \Sigma$-field over a $\sigma$-computable $\mathbb{K}$. For instance, for $\Sigma^{*}$ extensions there is the following result given in $[7$, Theorem 2.3] or [9, Corollary 2.2.4].

THEOREM 1. Let $(\mathbb{F}(t), \sigma)$ be a difference field extension of $(\mathbb{F}, \sigma)$. Then this is a $\Sigma^{*}$-extension iff $\sigma(t)=t+\beta, t \notin \mathbb{F}$, $\beta \in \mathbb{F}$, and there is no $g \in \mathbb{F}$ with $\sigma(g)-g=\beta$.

In particular, this result states that indefinite summation and building up $\Sigma^{*}$-extensions are closely related. Namely, if one fails to find a $g \in \mathbb{F}$ with $\sigma(g)-g=\beta \in \mathbb{F}$, i.e., one cannot solve the telescoping problem in $\mathbb{F}$, one can adjoin the solution $t$ with $\sigma(t)+t=\beta$ to $\mathbb{F}$ in form of the $\Sigma^{*}$-extension $(\mathbb{F}(t), \sigma)$ of $(\mathbb{F}, \sigma)$.

Our refined simplification strategy for a given sum is as follows: If we fail to solve the telescoping problem, we do not adjoin immediately the sum in form of a $\Sigma^{*}$-extension, but we first try to find an appropriate $\Pi \Sigma$-extension in which the sum can be formulated less nested. These ideas can be clarified further with the depth-function. Let $\mathbb{F}=\mathbb{K}\left(t_{1}, \ldots, t_{e}\right)$ be a function field over $\mathbb{K}$. Then for $g=\frac{g_{1}}{g_{2}} \in \mathbb{F}^{*}$ with $g_{i} \in \mathbb{K}\left[t_{1}, \ldots, t_{e}\right]$ and $\operatorname{gcd}_{\mathbb{K}\left[t_{1}, \ldots, t_{e}\right]}\left(g_{1}, g_{2}\right)=1$ we define the support of $g$, in short $\operatorname{supp}_{\mathbb{F}}(g)$, as those $t_{i}$ that occur in $g_{1}$ or $g_{2}$. Then for a $\Pi \Sigma$-field $(\mathbb{F}, \sigma)$ over $\mathbb{K}$ with $\mathbb{F}:=\mathbb{K}\left(t_{1}\right) \ldots\left(t_{e}\right)$ and $\sigma\left(t_{i}\right)=\alpha_{i} t_{i}+\beta_{i}$ for $\alpha_{i}, \beta_{i} \in \mathbb{K}\left(t_{1}\right) \ldots\left(t_{i-1}\right)$, the depthfunction depth $: \mathbb{F} \rightarrow \mathbb{N}_{0}$ is defined recursively as follows. For any $g \in \mathbb{K}$ set $\operatorname{depth}(g)=0$. If the depth-function is defined for $\left(\mathbb{K}\left(t_{1}\right) \ldots\left(t_{i-1}\right), \sigma\right)$ with $i>1$, we first define $\operatorname{depth}\left(t_{i}\right)=\max \left(\operatorname{depth}\left(\alpha_{i}\right), \operatorname{depth}\left(\beta_{i}\right)\right)+1$ and then define $\operatorname{depth}(g)=\max \left(\left\{\operatorname{depth}(x) \mid x \in \operatorname{supp}_{\mathbb{K}\left(t_{1}, \ldots, t_{i}\right)}(g)\right\} \cup\{0\}\right)$ for any $g \in \mathbb{K}\left(t_{1}\right) \ldots\left(t_{i}\right)$. The depth of $(\mathbb{F}, \sigma)$, in $\operatorname{short} \operatorname{depth}(\mathbb{F})$, is the maximal depth of all elements in $\mathbb{F}$, i.e., $\operatorname{depth}(\mathbb{F})$ is equal to $\max \left(0, \operatorname{depth}\left(t_{1}\right), \ldots, \operatorname{depth}\left(t_{e}\right)\right)$. We say that a $\Pi \Sigma / \Sigma^{*}$-extension $\left(\mathbb{F}\left(t_{1}\right) \ldots\left(t_{e}\right), \sigma\right)$ of a $\Pi \Sigma$-field $(\mathbb{F}, \sigma)$ has maximal depth $d$ if $\operatorname{depth}\left(t_{i}\right) \leq d$ for all $1 \leq i \leq e$.

Now we can reformulate Problem $R S$ as follows. Given a $\Pi \Sigma$-field $(\mathbb{F}, \sigma)$ with depth $d$ and $f \in \mathbb{F}^{n}$. Decide constructively if there is a single-nested $\Pi \Sigma$-extension $(\mathbb{E}, \sigma)$ of $(\mathbb{F}, \sigma)$ 
with maximal depth $d, g \in \mathbb{E}$ and $\mathbf{0} \neq \boldsymbol{c} \in \mathbb{K}^{n}$ such that $\sigma(g)-g=\boldsymbol{c} \boldsymbol{f}$.

Example 1. Denote the left hand side in (1) with $S_{n}^{(3)}$ and define $S_{n}^{(1)}:=\sum_{i=1}^{n} \frac{1}{i}$ and $S_{n}^{(2)}:=\sum_{j=1}^{n} S_{j}^{(1)} / j$. In the straightforward summation approach one applies usual telescoping which results in the $\Pi \Sigma$-field $\left(\mathbb{Q}\left(t_{1}\right)\left(t_{2}\right)\left(t_{3}\right)\left(t_{4}\right), \sigma\right)$ over $\mathbb{Q}$ with $\sigma\left(t_{1}\right)=t_{1}+1, \sigma\left(t_{2}\right)=t_{2}+\frac{1}{t_{1}+1}, \sigma\left(t_{3}\right)=$ $t_{3}+\sigma\left(\frac{t_{2}}{t_{1}}\right)$ and $\sigma\left(t_{4}\right)=t_{4}+\sigma\left(\frac{t_{3}}{t_{1}}\right)$, i.e., there is no $g \in$ $\mathbb{Q}\left(t_{1}\right)$ with $\sigma(g)-g=\frac{1}{t_{1}+1}$ and no $g \in \mathbb{Q}\left(t_{1}\right) \ldots\left(t_{r}\right)$ with $\sigma(g)-g=\sigma\left(\frac{t_{r}}{t_{1}}\right)$ for $r=2,3$. Then $t_{r}$ represents $S_{n}^{(r-1)}$ with $\operatorname{depth}\left(t_{r}\right)=r$ for $r=2,3,4$, and $\operatorname{depth}\left(\mathbb{Q}\left(t_{1}\right) \ldots\left(t_{4}\right)\right)=4$. But with our refined summation approach we obtain the following improvement starting from the $\Pi \Sigma$-field $(\mathbb{F}, \sigma)$ with $\mathbb{F}:=\mathbb{Q}\left(t_{1}\right)\left(t_{2}\right)$. We find the $\Sigma^{*}$-extension $(\mathbb{F}(s), \sigma)$ of $(\mathbb{F}, \sigma)$ with $\sigma(s)=s+\frac{1}{\left(t_{1}+1\right)^{2}}$ with the solution $g:=\frac{t_{2}^{2}+s}{2}$ for $\sigma(g)-g=\sigma\left(\frac{t_{2}}{t_{1}}\right)$ that represents the sum $S_{n}^{(2)}$. Moreover, we find the $\Sigma^{*}$-extension $\left.\left(\mathbb{F}(s)\left(s^{\prime}\right)\right), \sigma\right)$ of $(\mathbb{F}(s), \sigma)$ with $\sigma\left(s^{\prime}\right)=s^{\prime}+\frac{1}{\left(t_{1}+1\right)^{3}}$ and the solution $g^{\prime}=\frac{1}{6}\left(t_{2}^{3}+3 t_{2} s+2 s^{\prime}\right)$ for $\sigma\left(g^{\prime}\right)-g^{\prime}=\sigma\left(\frac{g}{t_{1}}\right)$. Then $S_{n}^{(3)}$ is represented by $g^{\prime}$ with $\operatorname{depth}\left(g^{\prime}\right)=2$ which gives the right hand side of identity (1).

Besides refined indefinite summation, we obtain a generalized version of creative telescoping in $\Pi \Sigma$-fields. Suppose that the sequences $f^{\prime}(m+i-1, k)$ can be represented with $f_{i} \in \mathbb{F}$ for $i \geq 1$ in a $\Pi \Sigma$-field $(\mathbb{F}, \sigma)$ over $\mathbb{K}(m)$ with $\operatorname{depth}\left(f_{i}\right)=d$. Moreover assume that we do not find a $g \in \mathbb{F}$ and $\mathbf{0} \neq \boldsymbol{c} \in \mathbb{K}(m)^{n}$ with $\sigma(g)-g=\boldsymbol{c} \boldsymbol{f}$ for $\boldsymbol{f}=\left(f_{1}, \ldots, f_{n}\right)$. Then the usual strategy is to increase $n$, i.e., the order of the possibly resulting creative telescoping recurrence. But if we find a solution for Problem $R S$, we derive a recurrence of order $n-1$ in terms of sum extensions with maximal depth $d$. Summarizing, for telescoping and creative telescoping we are interested in finding a single-nested $\Pi \Sigma$-extension in which a nontrivial linear combination of $\left(f_{1}, \ldots, f_{n}\right)$ in the solution space exists. More generally, we will ask for those extensions that will give us additional linear combinations. To make this more precise, we define for any $\mathbb{A} \subseteq \mathbb{F}^{n+1}$ the set $\Pi_{n}(\mathbb{A}):=\left\{\left(a_{1}, \ldots, a_{n}\right) \mid\left(a_{1}, \ldots, a_{n}, a_{n+1}\right) \in \mathbb{A}\right\}$.

Definition 1. Let $(\mathbb{E}, \sigma)$ be a $\Pi \Sigma$-field over $\mathbb{K}$ with depth $d, 1 \leq \delta \leq d+1$, and $\boldsymbol{f} \in \mathbb{E}^{n}$. We call a $\Pi \Sigma$-extension $(\mathbb{G}, \sigma)$ of $(\mathbb{E}, \sigma)$ single-nested $\delta$-complete for $\boldsymbol{f}$ if for all single-nested $\Pi \Sigma$-extensions $(\mathbb{H}, \sigma)$ of $(\mathbb{E}, \sigma)$ with maximal depth $\delta$ we have

$$
\Pi_{n}(\mathrm{~V}((1,-1), \boldsymbol{f}, \mathbb{H})) \subseteq \Pi_{n}(\mathrm{~V}((1,-1), \boldsymbol{f}, \mathbb{G})) .
$$

In this paper we solve the following problem. Given a $\Pi \Sigma$ field $(\mathbb{E}, \sigma)$ over a $\sigma$-computable $\mathbb{K}$ with depth $d, f \in \mathbb{E}^{n}$ and $\delta \in \mathbb{N}$ with $1 \leq \delta \leq d+1$; compute a single-nested $\Sigma^{*}$-extension $(\mathbb{G}, \sigma)$ of $(\mathbb{E}, \sigma)$ with maximal depth $\delta$ which is single-nested $\delta$-complete for $\boldsymbol{f}$, and compute a basis of $\mathrm{V}((1,-1), \boldsymbol{f}, \mathbb{G})$. Note that Problem $R S$ for single-nested $\Pi \Sigma$-extension is contained in this problem by setting $\delta:=d$.

\section{A MORE GENERAL PROBLEM}

In order to treat the problem stated in the previous paragraph, we solve the more general problem to find an $\mathbb{F}$ complete extension of $(\mathbb{E}, \sigma)$ for $\boldsymbol{f}$ defined in
Definition 2. Let $(\mathbb{E}, \sigma)$ be a $\Pi \Sigma$-extension of $(\mathbb{F}, \sigma)$ and $f \in \mathbb{E}^{n}$. We call a $\Pi \Sigma$-extension $(\mathbb{G}, \sigma)$ of $(\mathbb{E}, \sigma)$ singlenested $\mathbb{F}$-complete for $\boldsymbol{f}$, or in short $\mathbb{F}$-complete for $\boldsymbol{f}$, if (2) holds for all $\Pi \Sigma$-extensions $(\mathbb{H}, \sigma)$ of $(\mathbb{E}, \sigma)$ over $\mathbb{F}$.

The following lemma is crucial in order to show in Theorem 2 that there exists a $\Sigma^{*}$-extension of $(\mathbb{E}, \sigma)$ over $\mathbb{F}$ which is $\mathbb{F}$-complete for $\boldsymbol{f}$. This means that it suffices to restrict to $\Sigma^{*}$-extensions. Moreover this lemma is needed to prove Theorem 6 which gives us the essential idea how one can compute such $\mathbb{F}$-complete extensions.

Lemma 1. Let $(\mathbb{E}, \sigma)$ be a $\Pi \Sigma$-extension of $(\mathbb{F}, \sigma)$ and $f \in$ $\mathbb{E}^{*}$. If there exists a single-nested $\Pi \Sigma$-extension $(\mathbb{G}, \sigma)$ of $(\mathbb{E}, \sigma)$ over $\mathbb{F}$ with a $g \in \mathbb{G} \backslash \mathbb{E}$ such that $\sigma(g)-g=f$ then there exists a $\Sigma^{*}$-extension $(\mathbb{E}(s), \sigma)$ of $(\mathbb{E}, \sigma)$ over $\mathbb{F}$ with a $w \in \mathbb{E}$ such that $\sigma(s+w)-(s+w)=f$.

Proof. Let $(\mathbb{G}, \sigma)$ be a $\Pi \Sigma$-extension of $(\mathbb{E}, \sigma)$ over $\mathbb{F}$, i.e., $\mathbb{G}=\mathbb{E}\left(t_{1}\right) \ldots\left(t_{e}\right)$ with $\sigma\left(t_{i}\right)=\alpha_{i} t_{i}+\beta_{i}$ and $\alpha_{i}, \beta_{i} \in \mathbb{F}$, and suppose that there is a $g \in \mathbb{G} \backslash \mathbb{E}$ with $\sigma(g)-g=f$. Then by Karr's Fundamental Theorem [6, Theorem 24], see also [7, Section 4], it follows that $g=\sum_{i=0}^{e} c_{i} t_{i}+w$ for some $w \in \mathbb{E}$ and $c_{i} \in \mathbb{K}$, where $c_{i}=0$ if $\sigma\left(t_{i}\right)-t_{i} \notin \mathbb{F}$. In particular, $\mathbf{0} \neq$ $\left(c_{1}, \ldots, c_{e}\right)$, since $g \notin \mathbb{E}$. Now let $\mathbb{E}(s)$ be a rational function field and suppose that the difference field extension $(\mathbb{E}(s), \sigma)$ of $(\mathbb{E}, \sigma)$ with $\sigma(s)-s=\sum_{i=1}^{e} c_{i}\left(\sigma\left(t_{i}\right)-t_{i}\right)=: \beta \in \mathbb{F}$ is not a $\Sigma^{*}$-extension. Then by Theorem 1 we can take a $g^{\prime} \in \mathbb{E}$ with $\sigma\left(g^{\prime}\right)-g^{\prime}=\beta$. Let $j$ be maximal such that $c_{j} \neq 0$. Then we have $\sigma(v)-v=\sigma\left(t_{j}\right)-t_{j} \in \mathbb{F}$ for $v:=\frac{1}{c_{j}}\left(g^{\prime}-\sum_{i=1}^{j-1} c_{i} t_{i}\right) \in$ $\mathbb{E}\left(t_{1}\right) \ldots\left(t_{j-1}\right)$, and thus $\left(\mathbb{E}\left(t_{1}\right) \ldots\left(t_{j-1}\right)\left(t_{j}\right), \sigma\right)$ is not a $\Sigma^{*}$ extension of $\left(\mathbb{E}\left(t_{1}\right) \ldots\left(t_{j-1}\right), \sigma\right)$ by Theorem 1 , a contradiction. Hence $(\mathbb{E}(s), \sigma)$ is a $\Sigma^{*}$-extension of $(\mathbb{E}, \sigma)$ over $\mathbb{F}$, and $\sigma(s+w)-(s+w)=\sum_{i=1}^{e} c_{i}\left(\sigma\left(t_{i}\right)-t_{i}\right)+\sigma(w)-w=$ $\sigma(g)-g=f$.

Observe that Lemma 1 follows immediately by Theorem 1 if one restricts to the special case $\mathbb{E}=\mathbb{F}$. For the case $\mathbb{F} \subsetneq \mathbb{E}$, in which we are actually interested, we needed Karr's Fundamental Theorem [6].

ThEOREM 2. Let $(\mathbb{E}, \sigma)$ be a $\Pi \Sigma$-extension of $(\mathbb{F}, \sigma)$ and $f \in \mathbb{E}^{n}$. Then there is a $\Sigma^{*}$-extension of $(\mathbb{E}, \sigma)$ over $\mathbb{F}$ which is $\mathbb{F}$-complete for $\boldsymbol{f}$.

Proof. Let $(\mathbb{G}, \sigma)$ be a $\Sigma^{*}$-extension of $(\mathbb{E}, \sigma)$ over $\mathbb{F}$ which is not $\mathbb{F}$-complete for $\boldsymbol{f}$. Then we can take a $\boldsymbol{c} \in \mathbb{K}^{n}$ such that $\sigma(g)-g=\boldsymbol{c} \boldsymbol{f} \in \mathbb{E}$ has a solution in some $\Pi \Sigma$ extension of $(\mathbb{E}, \sigma)$ over $\mathbb{F}$, but no solution in $\mathbb{E}$. Then by Lemma 1 it follows that there is a $\Sigma^{*}$-extension $(\mathbb{E}(s), \sigma)$ of $(\mathbb{E}, \sigma)$ over $\mathbb{F}$ with $\sigma(s+w)-(s+w)=f$ for some $w \in \mathbb{E}$. Observe that there also does not exist an $h \in$ $\mathbb{G}$ with $\sigma(h)-h=\beta \in \mathbb{F}$. Otherwise we would have $\sigma(h+w)-(h+w)=\boldsymbol{c} \boldsymbol{f}$ with $h+w \in \mathbb{G}$, a contradiction. Consequently, by Theorem 1 also $(\mathbb{G}(s), \sigma)$ is a $\Sigma^{*}$-extension of $(\mathbb{G}, \sigma)$ with $\sigma(s)=s+\beta$ and therefore a $\Sigma^{*}$-extension of $(\mathbb{E}, \sigma)$ over $\mathbb{F}$. Since $\Pi_{n}(\mathrm{~V}((1,-1), \boldsymbol{f}, \mathbb{G}))$ is a proper subspace of $\Pi_{n}(\mathrm{~V}((1,-1), \boldsymbol{f}, \mathbb{G}(s)))$ and those spaces have dimension at most $n$, this argument can be repeated at most $n$ times before an $\mathbb{F}$-complete $\Sigma^{*}$-extension is reached.

In the following we will represent the $\Pi \Sigma$-field $(\mathbb{E}, \sigma)$ in such a way that one can find a single-nested $\delta$-complete extension of $(\mathbb{E}, \sigma)$ for $\boldsymbol{f}$ by finding an $\mathbb{F}$-complete extension over a certain subfield $\mathbb{F} \subseteq \mathbb{E}$. 
The crucial idea is that the generators of a $\Pi \Sigma$-extension $\left(\mathbb{F}\left(t_{1}\right) \ldots\left(t_{e}\right), \sigma\right)$ of $(\mathbb{F}, \sigma)$ can be reordered by increasing depth without changing the $\Pi \Sigma$ nature of the extension; this is trivial since no term can depend on terms of higher depth. For further details we refer to [14].

Hence one can reorder a $\Pi \Sigma$-field $(\mathbb{E}, \sigma)$ over $\mathbb{K}$ with depth $d$ and $1 \leq \delta \leq d+1$ to a $\Pi \Sigma$-field $\left(\mathbb{F}\left(t_{1}\right) \ldots\left(t_{e}\right), \sigma\right)$ with $\operatorname{depth}(\mathbb{F})=\delta-1$ and $\operatorname{depth}\left(t_{i}\right) \geq \delta$ for all $1 \leq i \leq e$. With this reordered $\Pi \Sigma$-field one obtains

Lemma 2. Let $\left(\mathbb{F}\left(t_{1}\right) \ldots\left(t_{e}\right), \sigma\right)$ be a $\Pi \Sigma$-field with $\delta:=$ $\operatorname{depth}(\mathbb{F})+1$ and $\operatorname{depth}\left(t_{i}\right) \geq \delta$ for $1 \leq i \leq e$, and let $(\mathbb{H}, \sigma)$ be a single-nested $\Pi \Sigma$-extension of $\left(\mathbb{F}\left(t_{1}\right) \ldots\left(t_{e}\right), \sigma\right)$. Then this extension has maximal depth $\delta$ iff it is over $\mathbb{F}$.

Proof. Write $\mathbb{H}:=\mathbb{F}\left(t_{1}\right) \ldots\left(t_{e}\right)\left(s_{1}\right) \ldots\left(s_{u}\right)$. First assume that the extension is over $\mathbb{F}$, i.e., $\sigma\left(s_{i}\right)=\alpha_{i} s_{i}+\beta_{i}$ with $\alpha_{i}, \beta_{i} \in \mathbb{F}$. Then, because of $\operatorname{depth}(\mathbb{F})=\delta-1$ it follows that $\operatorname{depth}\left(\beta_{i}\right) \leq \delta-1$ and $\operatorname{depth}\left(\alpha_{i}\right) \leq \delta-1$, thus $\operatorname{depth}\left(s_{i}\right)=\max \left(\operatorname{depth}\left(\alpha_{i}\right), \operatorname{depth}\left(\beta_{i}\right)\right)+1 \leq \delta$, and therefore the extension has maximal depth $\delta$. Conversely, suppose that this extension has maximal depth $\delta$, i.e. $\operatorname{depth}\left(s_{i}\right) \leq \delta$. Then $\operatorname{depth}\left(\alpha_{i}\right) \leq \delta-1$ and $\operatorname{depth}\left(\beta_{i}\right) \leq \delta-1$, and consequently $\alpha_{i}, \beta_{i} \in \mathbb{F}$.

TheOREM 3. Let $(\mathbb{E}, \sigma)$ with $\mathbb{E}:=\mathbb{F}\left(t_{1}\right) \ldots\left(t_{e}\right)$ be a $\Pi \Sigma$ field where $\delta:=\operatorname{depth}(\mathbb{F})+1$ and $\operatorname{depth}\left(t_{i}\right) \geq \delta$ for $1 \leq i \leq e$, and $\boldsymbol{f} \in \mathbb{E}^{n}$. Then a $\Pi \Sigma$-extension $(\mathbb{G}, \sigma)$ of $(\mathbb{E}, \sigma)$ over $\mathbb{F}$ which is $\mathbb{F}$-complete for $\boldsymbol{f}$ has maximal depth $\delta$ and is singlenested $\delta$-complete for $\boldsymbol{f}$.

Proof. Assume such an extension $(\mathbb{G}, \sigma)$ of $(\mathbb{E}, \sigma)$ is not single-nested $\delta$-complete for $\boldsymbol{f}$. Then take a single-nested $\Pi \Sigma$-extension $(\mathbb{H}, \sigma)$ of $(\mathbb{E}, \sigma)$ with maximal depth $\delta$ and $\boldsymbol{c} \in \Pi_{n}(\mathrm{~V}((1,-1), \boldsymbol{f}, \mathbb{H})) \backslash \Pi_{n}(\mathrm{~V}((1,-1), \boldsymbol{f}, \mathbb{G}))$. Since $\delta=$ $\operatorname{depth}(\mathbb{F})+1$ and $\operatorname{depth}\left(t_{i}\right) \geq \delta,(\mathbb{H}, \sigma)$ is an extension of $(\mathbb{E}, \sigma)$ over $\mathbb{F}$ by Lemma 2 , and thus the extension $(\mathbb{G}, \sigma)$ of $(\mathbb{E}, \sigma)$ is not $\mathbb{F}$-complete for $\boldsymbol{f}$, a contradiction. Moreover, the extension $(\mathbb{G}, \sigma)$ of $(\mathbb{E}, \sigma)$ is single-nested with maximal depth $\delta$ by Lemma 2 .

In Section 5 we will develop an algorithm that computes an $\mathbb{F}$-complete $\Sigma^{*}$-extension of $\left(\mathbb{F}\left(t_{1}\right) \ldots\left(t_{e}\right), \sigma\right)$ over $\mathbb{F}$ for $\boldsymbol{f}$. Then by Theorem 3 this extension will be also single-nested $\delta$-complete for $\boldsymbol{f}$ with maximal depth $\delta$.

\section{A REDUCTION STRATEGY}

We develop a streamlined version of Karr's summation algorithm [6] based on results of [2] and [9, 12, 10, 11] that solves Problem PFLDE. In particular, this approach will assist in finding $\mathbb{F}$-complete extensions over $\mathbb{F}$.

More precisely, let $(\mathbb{F}(t), \sigma)$ be a $\Pi \Sigma$-extension of $(\mathbb{F}, \sigma)$ with $\sigma(t)=\alpha t+\beta, \mathbb{K}=$ const $_{\sigma} \mathbb{F}, \mathbf{0} \neq \boldsymbol{a}=\left(a_{1}, a_{2}\right) \in \mathbb{F}(t)^{2}$ and $\boldsymbol{f} \in \mathbb{F}(t)^{n}$. We will introduce a simplified version of Karr's reduction strategy [6] that helps in finding a basis of $\mathrm{V}(\boldsymbol{a}, \boldsymbol{f}, \mathbb{F}(t))$ over $\mathbb{K}$. If $(\mathbb{F}, \sigma)$ is a $\Pi \Sigma$-field, this reduction turns into a complete algorithm. Moreover, this reduction technique will deliver all the information to compute an $\mathbb{F}$ complete extension.

A special case. If $a_{1} a_{2}=0$, we have $\boldsymbol{g}=\boldsymbol{c} \sigma^{-1}\left(\frac{\boldsymbol{f}}{a_{1}}\right)$ with $a_{1} \neq 0$ or $\boldsymbol{g}=\boldsymbol{c} \frac{\boldsymbol{f}}{a_{2}}$ with $a_{2} \neq 0$. Then it follows with $\boldsymbol{g}=\left(g_{1}, \ldots, g_{n}\right)$ and the $i$-th unit vector $(0 \ldots, 1, \ldots, 0) \in$ $\mathbb{K}^{n}$ that $\left\{\left(0 \ldots, 1, \ldots, 0, g_{i}\right)\right\}_{1<i<n} \subseteq \mathbb{K}^{n} \times \mathbb{F}(t)$ is a basis of $\mathrm{V}(\boldsymbol{a}, \boldsymbol{f}, \mathbb{F}(t))$. Hence from now on we suppose $\boldsymbol{a} \in\left(\mathbb{F}(t)^{*}\right)^{2}$.
Clearing denominators/cancelling common factors. Compute $\boldsymbol{a}^{\prime}=\left(a_{1}^{\prime}, a_{2}^{\prime}\right) \in\left(\mathbb{F}[t]^{*}\right)^{2}, \boldsymbol{f}^{\prime}=\left(f_{1}^{\prime}, \ldots, f_{n}^{\prime}\right) \in$ $\mathbb{F}[t]^{n}$ such that $\operatorname{gcd}_{\mathbb{F}[t]}\left(f_{1}^{\prime}, \ldots, f_{n}^{\prime}, a_{1}^{\prime}, a_{2}^{\prime}\right)=1$ and $\boldsymbol{a}^{\prime}=\boldsymbol{a} q$, $\boldsymbol{f}^{\prime}=\boldsymbol{f} q$ for some $q \in \mathbb{F}(t)^{*}$. Then we have $\mathrm{V}(\boldsymbol{a}, \boldsymbol{f}, \mathbb{F}(t))=$ $\mathrm{V}\left(\boldsymbol{a}^{\prime}, \boldsymbol{f}^{\prime}, \mathbb{F}(t)\right)$. Therefore we may suppose that $\boldsymbol{a} \in\left(\mathbb{F}[t]^{*}\right)^{2}$ and $f \in \mathbb{F}[t]^{n}$ where the entries have no common factors.

In Karr's original approach [6] the solutions $g=p+q \in$ $\mathbb{F}(t)$ in $\left(c_{1}, \ldots, c_{n}, g\right) \in \mathrm{V}(\boldsymbol{a}, \boldsymbol{f}, \mathbb{F}(t))$ are computed by deriving first the polynomial part $p \in \mathbb{F}[t]$ and afterwards the fractional part $q \in \mathbb{F}(t)$, i.e., the degree of the numerator is smaller than the degree of the denominator. We simplify this approach substantially by first computing a common denominator of all the possible solutions in $\mathbb{F}(t)$ and afterwards computing the numerator of the solutions over this common denominator.

Denominator bounding. In the first important reduction we bound the possible denominators of the solution space $\mathrm{V}(\boldsymbol{a}, \boldsymbol{f}, \mathbb{F}(t))$. More precisely, we look for a denominator bound $d$ of $\mathrm{V}(\boldsymbol{a}, \boldsymbol{f}, \mathbb{F}(t))$, i.e., a polynomial $d \in \mathbb{F}[t]^{*}$ that satisfies

$$
\forall\left(c_{1}, \ldots, c_{n}, g\right) \in \mathrm{V}(\boldsymbol{a}, \boldsymbol{f}, \mathbb{F}(t)): d g \in \mathbb{F}[t] .
$$

Since $\mathrm{V}(\boldsymbol{a}, \boldsymbol{f}, \mathbb{F}(t))$ is a finite dimensional vector space over $\mathbb{K}$, a denominator bound must exist. Now suppose that we have given such a $d$ and define $\boldsymbol{a}^{\prime}:=\left(\frac{a_{1}}{\sigma(d)}, \frac{a_{2}}{d}\right)$. Then it follows that $\left\{\left(c_{i 1}, \ldots, c_{i n}, g_{i}\right)\right\}_{1 \leq i \leq r}$ is a basis of $\mathrm{V}\left(\boldsymbol{a}^{\prime}, \boldsymbol{f}, \mathbb{F}[t]\right)$ if and only if $\left\{\left(c_{i 1}, \ldots, c_{i n}, \frac{g_{i}}{d}\right)\right\}_{1 \leq i \leq r}$ is a basis of $\mathrm{V}(\boldsymbol{a}, \boldsymbol{f}, \mathbb{F}(t))$. For a proof we refer to $[9,12]$. Hence, given a denominator bound $d$ of $\mathrm{V}(\boldsymbol{a}, \boldsymbol{f}, \mathbb{F}(t))$, we can reduce the problem to search for a basis of $\mathrm{V}(\boldsymbol{a}, \boldsymbol{f}, \mathbb{F}(t))$ to look for a basis of $\mathrm{V}\left(\boldsymbol{a}^{\prime}, \boldsymbol{f}, \mathbb{F}[t]\right)$. By clearing denominators and cancelling common factors in $\boldsymbol{a}$ and $\boldsymbol{f}$, as above, we may also suppose that $\boldsymbol{a} \in\left(\mathbb{F}[t]^{*}\right)^{2}$ and $\boldsymbol{f} \in \mathbb{F}[t]^{n}$.

Polynomial degree bounding. The next step consists of bounding the polynomial degrees in $\mathrm{V}(\boldsymbol{a}, \boldsymbol{f}, \mathbb{F}[t])$. For convenience we introduce $\mathbb{F}[t]_{b}:=\{f \in \mathbb{F}[t] \mid \operatorname{deg}(f) \leq b\}$ for $b \in \mathbb{N}_{0}$ and $\mathbb{F}[t]_{-1}:=\{0\}$. Moreover, we define $\|b\|:=\operatorname{deg} b$ for $b \in \mathbb{F}[t]^{*},\|0\|:=-1$, and $\|\boldsymbol{b}\|:=\max _{1 \leq i \leq l}\left\|b_{i}\right\|$ for $\boldsymbol{b}=$ $\left(b_{1}, \ldots, b_{l}\right) \in \mathbb{F}[t]^{l}$. Then we look for a polynomial degree bound $b$ of $\mathrm{V}(\boldsymbol{a}, \boldsymbol{f}, \mathbb{F}[t])$, i.e., a $b \in \mathbb{N}_{0} \cup\{-1\}$ such that

$\mathrm{V}\left(\boldsymbol{a}, \boldsymbol{f}, \mathbb{F}[t]_{b}\right)=\mathrm{V}(\boldsymbol{a}, \boldsymbol{f}, \mathbb{F}[t]), b \geq \max (-1,\|\boldsymbol{f}\|-\|\boldsymbol{a}\|)$.

Again, since $\mathrm{V}(\boldsymbol{a}, \boldsymbol{f}, \mathbb{F}[t])$ is finite dimensional over $\mathbb{K}$, a degree bound must exist. Note that by the second condition in (3) it follows that $\boldsymbol{f} \in \mathbb{F}[t]_{\|\boldsymbol{a}\|+b}$ which is needed to proceed with the degree elimination technique below.

Due to $[6,7,2]$ the problem to determine a denominator bound or degree bound is completely constructive if $(\mathbb{F}, \sigma)$ is a $\Pi \Sigma$-field over a $\sigma$-computable $\mathbb{K}$. The proofs and subalgorithms of these results can be found in $[2,10,11]$.

TheOREm 4. Let $(\mathbb{F}(t), \sigma)$ be a $\Pi \Sigma$-field over a $\sigma$-computable $\mathbb{K}, \boldsymbol{a} \in\left(\mathbb{F}[t]^{*}\right)^{2}$ and $\boldsymbol{f} \in \mathbb{F}[t]^{n}$. Then there are algorithms that compute a denominator bound of $\mathrm{V}(\boldsymbol{a}, \boldsymbol{f}, \mathbb{F}(t))$ or a degree bound of $\mathrm{V}(\boldsymbol{a}, \boldsymbol{f}, \mathbb{F}[t])$.

Polynomial degree reduction. Finally we have to deal with the problem to compute a basis of $\mathrm{V}\left(\boldsymbol{a}, \boldsymbol{f}, \mathbb{F}[t]_{\delta}\right)$ for some $\delta \in \mathbb{N}_{0} \cup\{-1\}$ where $\boldsymbol{f} \in \mathbb{F}[t]_{\delta+l}^{n}$ with $l:=\|\boldsymbol{a}\|$. Here we follow exactly the idea in [6]. Namely, we first find the candidates of the leading coefficients $g_{\delta} \in \mathbb{F}$ for the solutions 
$\left(c_{1}, \ldots, c_{n}, g\right) \in \mathrm{V}\left(\boldsymbol{a}, \boldsymbol{f}, \mathbb{F}[t]_{\delta}\right)$ with $g=\sum_{i=0}^{\delta} g_{i} t^{i}$, plugging back its solution space and go on recursively to derive the candidates of the missing coefficients $g_{i} \in \mathbb{F}$. More precisely, define

$$
\begin{gathered}
\tilde{\boldsymbol{a}}_{\delta}=\left(\tilde{a}_{1}, \tilde{a}_{2}\right):=\left(\operatorname{coeff}\left(a_{1}, l\right) \alpha^{\delta}, \operatorname{coeff}\left(a_{2}, l\right)\right), \\
\tilde{\boldsymbol{f}}_{\delta}:=\left(\operatorname{coeff}\left(f_{1}, \delta+l\right), \ldots, \operatorname{coeff}\left(f_{n}, \delta+l\right)\right) .
\end{gathered}
$$

where $\mathbf{0} \neq \tilde{\boldsymbol{a}}_{\boldsymbol{\delta}} \in \mathbb{F}^{2}$ and $\tilde{\boldsymbol{f}}_{\boldsymbol{\delta}} \in \mathbb{F}^{n} ; \operatorname{coeff}(p, l)$ gives the $l$ th coefficient of $p \in \mathbb{F}[t]$. Then the right linear combinations of a basis of $\operatorname{V}\left(\tilde{\boldsymbol{a}}_{\boldsymbol{\delta}}, \tilde{\boldsymbol{f}}_{\boldsymbol{\delta}}, \mathbb{F}\right)$ enable one to construct partially the solutions $\left(c_{1}, \ldots, c_{n}, g\right) \in \mathrm{V}\left(\boldsymbol{a}, \boldsymbol{f}, \mathbb{F}[t]_{\delta}\right)$, namely $\left(c_{1}, \ldots, c_{n}\right) \in \mathbb{K}^{n}$ with the $\delta$-th coefficient $g_{\delta}$ in $g \in \mathbb{F}[t]_{\delta}$. Given this basis of $\mathrm{V}\left(\tilde{\boldsymbol{a}}_{\boldsymbol{\delta}}, \tilde{\boldsymbol{f}}_{\boldsymbol{\delta}}, \mathbb{F}\right)$, one plugs in the possible solutions for the leading coefficients, and ends up to find a basis of a solution space $\mathrm{V}\left(\boldsymbol{a}, \boldsymbol{f}_{\boldsymbol{\delta}-\mathbf{1}}, \mathbb{F}[t]_{\delta-1}\right)$ which contains all the information for the remaining coefficients. Details how this vector $\boldsymbol{f}_{\boldsymbol{\delta}-\boldsymbol{1}}$ with entries in $\mathbb{F}[t]_{\delta+l-1}$ can be computed are given in $[12,14]$. Combining the bases of these two solution spaces, i.e., the possible leading coefficients and its remaining coefficients, one can reconstruct a basis of the original solution space $\mathrm{V}\left(\boldsymbol{a}, \boldsymbol{f}, \mathbb{F}[t]_{\delta}\right)$. Applying this reduction technique recursively one has to deal with the problem to find bases of solution spaces $\mathrm{V}\left(\boldsymbol{a}, \boldsymbol{f}_{\boldsymbol{i}}, \mathbb{F}[t]_{i}\right)$ for $-1 \leq i \leq \delta-1$ where the $\boldsymbol{f}_{\boldsymbol{i}}$ have entries in $\mathbb{F}[t]_{i+l}$. This can be achieved by computing bases of the solution spaces $\mathrm{V}\left(\tilde{\boldsymbol{a}}_{\boldsymbol{i}}, \tilde{\boldsymbol{f}}_{\boldsymbol{i}}, \mathbb{F}\right)$ for $0 \leq i \leq \delta$ with $\tilde{\boldsymbol{a}}_{\boldsymbol{i}} \in \mathbb{F}^{2}$ and $\tilde{\boldsymbol{f}}_{\boldsymbol{i}} \in \mathbb{F}^{n}$, and by dealing with the following

Base case $I$. In the incremental reduction we finally reach the problem to find a basis of $\mathrm{V}\left(\boldsymbol{a}, \boldsymbol{f}, \mathbb{F}[t]_{-1}\right)$ with $\mathbb{F}[t]_{-1}=\{0\}$. Then we have $\mathrm{V}(\boldsymbol{a}, \boldsymbol{f},\{0\})=$ Nullspace $_{\mathbb{K}}(\boldsymbol{f}) \times\{0\}$ where Nullspace $_{\mathbb{K}}(\boldsymbol{f})=\left\{\boldsymbol{k} \in \mathbb{K}^{n} \mid \boldsymbol{f} \boldsymbol{k}=0\right\}$. Note that a basis of $\mathrm{V}(\boldsymbol{a}, \boldsymbol{f},\{0\})$ can be computed by linear algebra if $(\mathbb{F}, \sigma)$ is a $\Pi \Sigma$-field over a $\sigma$-computable $\mathbb{K}$; for more details see [12].

Summarizing, let $(\mathbb{F}(t), \sigma)$ be a $\Pi \Sigma$-extension of $(\mathbb{F}, \sigma)$, $\boldsymbol{a} \in\left(\mathbb{F}[t]^{*}\right)^{2}$ with $l:=\|\boldsymbol{a}\|$ and $\boldsymbol{f} \in \mathbb{F}[t]_{\delta+l}^{n}$ for some $\delta \in$ $\mathbb{N}_{0} \cup\{-1\}$. Then we can apply this reduction technique step by step and obtain an incremental reduction of $\left(\boldsymbol{a}, \boldsymbol{f}, \mathbb{F}[t]_{\delta}\right)$. We call $\left\{\left(\boldsymbol{a}, \boldsymbol{f}_{\boldsymbol{\delta}}, \mathbb{F}[t]_{\delta}\right), \ldots,\left(\boldsymbol{a}, \boldsymbol{f}_{-\mathbf{1}}, \mathbb{F}[t]_{-1}\right)\right\}$ the incremental tuples and $\left\{\left(\tilde{\boldsymbol{a}}_{\boldsymbol{\delta}}, \tilde{\boldsymbol{f}}_{\boldsymbol{\delta}}, \mathbb{F}\right), \ldots,\left(\tilde{\boldsymbol{a}}_{\mathbf{0}}, \tilde{\boldsymbol{f}}_{\mathbf{0}}, \mathbb{F}\right)\right\}$ the coefficient tuples of such an incremental reduction.

Example 2. Take the $\Pi \Sigma$-field $\left(\mathbb{Q}\left(t_{1}\right)\left(t_{2}\right), \sigma\right)$ over $\mathbb{Q}$ from Example 1, i.e., $\sigma\left(t_{1}\right)=t_{1}+1$ and $\sigma\left(t_{2}\right)=t_{2}+\frac{1}{t_{1}+1}$, and write $\mathbb{F}:=\mathbb{Q}\left(t_{1}\right)$. With our reduction strategy we will find a basis of $\mathrm{V}\left(\boldsymbol{a}, \boldsymbol{f}, \mathbb{F}\left(t_{2}\right)\right)$ for $\boldsymbol{a}=(1,-1) \in \mathbb{F}\left(t_{2}\right)^{2}$ and $\boldsymbol{f}=\left(\sigma\left(t_{2} / t_{1}\right)\right)=\left(\frac{1+\left(t_{1}+1\right) t_{2}}{\left(t_{1}+1\right)^{2}}\right) \in \mathbb{F}\left(t_{2}\right)^{1}$. Clearing denominators gives $\boldsymbol{a}=\left(\left(t_{1}+1\right)^{2},-\left(t_{1}+1\right)^{2}\right) \in \mathbb{F}\left[t_{2}\right]^{2}, \boldsymbol{f}=$ $\left(1+\left(t_{1}+1\right) t_{2}\right) \in \mathbb{F}\left[t_{2}\right]^{1}$. A den. bound of $\mathrm{V}\left(\boldsymbol{a}, \boldsymbol{f}, \mathbb{F}\left(t_{2}\right)\right)$ is 1 , and a degree bound of $\mathrm{V}\left(\boldsymbol{a}, \boldsymbol{f}, \mathbb{F}\left[t_{2}\right]\right)$ is 2 . Now we start the incremental reduction of $\left(\boldsymbol{a}, \boldsymbol{f}, \mathbb{F}\left[t_{2}\right]_{2}\right)$. For the incremental tuple $\left(\boldsymbol{a}, \boldsymbol{f}_{\mathbf{2}}, \mathbb{F}\left[t_{2}\right]_{2}\right)$ with $\boldsymbol{f}_{\mathbf{2}}:=\boldsymbol{f} \in \mathbb{F}\left[t_{2}\right]_{2}^{1}$ we obtain the coefficient tuple $(\boldsymbol{a},(0), \mathbb{F})$. The basis $\{(1,0),(0,1)\}$ of $\mathrm{V}(\boldsymbol{a},(0), \mathbb{F})$ gives us the incremental tuple $\left(\boldsymbol{a}, \boldsymbol{f}_{\mathbf{1}}, \mathbb{F}\left[t_{2}\right]_{1}\right)$ with $\boldsymbol{f}_{\mathbf{1}}=\left(1+\left(t_{1}+1\right) t_{2},-1-2\left(t_{1}+1\right) t_{2}\right) \in \mathbb{F}\left[t_{2}\right]_{1}^{2}$ and the coefficient tuple $\left(\boldsymbol{a},\left(t_{1}+1,-2\left(t_{1}+1\right)\right), \mathbb{F}\right)$. Then taking the basis $\{(2,1,0),(0,0,1)\}$ of $\mathrm{V}(\boldsymbol{a},(1,-2), \mathbb{F})$, one obtains $f_{0}=\left(1,-t_{1}-1\right) \in \mathbb{F}$, the incremental tuple $\left(\boldsymbol{a}, \boldsymbol{f}_{\mathbf{0}}, \mathbb{F}\left[t_{2}\right]_{0}\right)$ and the coefficient tuple $\left(\boldsymbol{a}, \boldsymbol{f}_{\mathbf{0}}, \mathbb{F}\right)$. A basis of $\mathrm{V}\left(\boldsymbol{a}, \boldsymbol{f}_{\mathbf{0}}, \mathbb{F}\right)$ is $\{(0,0,1)\}$ which defines $\boldsymbol{f}_{-\mathbf{1}}=(0)$. Finally, we end up in the base case $\mathrm{V}\left(\boldsymbol{a}, \boldsymbol{f}_{-\mathbf{1}},\{0\}\right)$ which immediately allows us to compute the basis $\{(1,0)\}$. Finally we can reconstruct the bases $\{(0,0,1)\}$ of $\mathrm{V}\left(\boldsymbol{a}, \boldsymbol{f}_{\boldsymbol{i}}, \mathbb{F}\left[t_{2}\right]_{i}\right)$ for $i \in\{0,1\}$ and therefore the basis $\{(0,1)\}$ of $\mathrm{V}\left(\boldsymbol{a}, \boldsymbol{f}_{\mathbf{2}}, \mathbb{F}\left[t_{2}\right]_{2}\right)$ and $\mathrm{V}\left(\boldsymbol{a}, \boldsymbol{f}, \mathbb{F}\left(t_{2}\right)\right)$. For further details we refer to $[12,14]$.

A reduction to $\mathbb{F}$. Suppose that we have given not only a single but a nested $\Pi \Sigma$-extension $\left(\mathbb{F}\left(t_{1}\right) \ldots\left(t_{e}\right), \sigma\right)$ of $(\mathbb{F}, \sigma)$ where we write $\mathbb{F}_{i}:=\mathbb{F}\left(t_{1}\right) \ldots\left(t_{i}\right)$ for $0 \leq i \leq e$, i.e., $\mathbb{F}_{0}=\mathbb{F}$. Let $\mathbf{0} \neq \boldsymbol{a}=\left(a_{1}, a_{2}\right) \in \mathbb{F}_{e}$ and $\overline{\boldsymbol{f}} \in \mathbb{F}_{e}^{n}$. Then we understand by a reduction of $\left(\boldsymbol{a}, \boldsymbol{f}, \mathbb{F}_{e}\right)$ to $\mathbb{F}$ a recursive application of the above reductions. More precisely, if $e=0$, we do nothing. Otherwise, suppose that $e>0$. If $a_{1} a_{2}=0$, we just apply the special case from above. Otherwise, within our reduction there is a denominator bound $d \in \mathbb{F}_{e-1}\left[t_{e}\right]^{*}$ which reduces the problem to find a basis of $\mathrm{V}\left(\boldsymbol{a}, \boldsymbol{f}, \mathbb{F}_{e}\right)$ to find one for $\mathrm{V}\left(\boldsymbol{a}^{\prime}, \boldsymbol{f}^{\prime}, \mathbb{F}_{e-1}\left[t_{e}\right]\right)$ for some $\boldsymbol{a}^{\prime} \in\left(\mathbb{F}_{e-1}\left[t_{e}\right]^{*}\right)^{2}$ and $\boldsymbol{f}^{\prime} \in \mathbb{F}_{e-1}\left[t_{e}\right]^{n}$; those are given by setting $\boldsymbol{a}^{\prime}:=\left(a_{1} / \sigma(d), a_{2} / d\right), \boldsymbol{f}^{\prime}:=\boldsymbol{f}$, and clearing denominators and cancelling common factors. Next, with a degree bound $b$ of $\mathrm{V}\left(\boldsymbol{a}^{\prime}, \boldsymbol{f}^{\prime}, \mathbb{F}_{e-1}\left[t_{e}\right]\right)$ the incremental reduction of $\left(\boldsymbol{a}^{\prime}, \boldsymbol{f}^{\prime}, \mathbb{F}_{e-1}\left[t_{e}\right]_{b}\right)$ is applied. Within this reduction the coefficient tuples $\left(\boldsymbol{a}_{\boldsymbol{i}}, \boldsymbol{f}_{\boldsymbol{i}}, \mathbb{F}_{e-1}\right)$ for $0 \leq i \leq b$ give the subreductions of $\left(\boldsymbol{a}_{\boldsymbol{i}}, \boldsymbol{f}_{\boldsymbol{i}}, \mathbb{F}_{e-1}\right)$ to $\mathbb{F}$ for $0 \leq i \leq b$ that define recursively the whole reduction of $\left(\boldsymbol{a}, \boldsymbol{f}, \overline{\mathbb{F}}_{e}\right)$ to $\mathbb{F}$.

We call $T$ the tuple set of a reduction of $\left(\boldsymbol{a}, \boldsymbol{f}, \mathbb{F}_{e}\right)$ to $\mathbb{F}$ if besides $\left(\boldsymbol{a}, \boldsymbol{f}, \mathbb{F}_{e}\right) \in T$ the set $T$ contains exactly all those coefficient tuples that occur in the recursively applied incremental reductions. Moreover, for $a_{e}:=a$ and $f_{e}:=f$ we call $\left\{\left(\boldsymbol{a}_{\boldsymbol{i}}, \boldsymbol{f}_{\boldsymbol{i}}, \mathbb{F}_{i}\right)\right\}_{r \leq i \leq e} \subseteq T$ path-tuples of $\left(\boldsymbol{a}_{\boldsymbol{r}}, \boldsymbol{f}_{\boldsymbol{r}}, \mathbb{F}_{r}\right) \in T$ if in the subreduction of $\left(\boldsymbol{a}_{\boldsymbol{i}+\mathbf{1}}, \boldsymbol{f}_{\boldsymbol{i}+\mathbf{1}}, \mathbb{F}_{i+1}\right)$ to $\mathbb{F}$ the coefficient tuple $\left(\boldsymbol{a}_{\boldsymbol{i}}, \boldsymbol{f}_{\boldsymbol{i}}, \mathbb{F}_{i}\right)$ occurs for each $r \leq i<e$ in the incremental reduction. Finally, we introduce the $\mathbb{F}_{r}$-critical tuple set $S$ in a reduction of $\left(\boldsymbol{a}, \boldsymbol{f}, \mathbb{F}_{e}\right)$ to $\mathbb{F}$ as that subset of the tuple set $T$ of the reduction to $\mathbb{F}$ that contains all $\left(\boldsymbol{a}^{\prime}, \boldsymbol{f}^{\prime}, \mathbb{F}_{r}\right) \in T$ with the following property: for its pathtuples $\left\{\left(\boldsymbol{a}_{\boldsymbol{i}}, \boldsymbol{f}_{\boldsymbol{i}}, \mathbb{F}_{i}\right)\right\}_{r \leq i \leq e}$ we have that $\boldsymbol{a}_{\boldsymbol{i}}$ is homogeneous for all $r \leq i \leq e$.

An algorithm. If the denominator bound problem and polynomial degree bound problem can be solved in the $\Pi \Sigma$ extensions $\left(\mathbb{F}_{i}, \sigma\right)$ of $\left(\mathbb{F}_{i-1}, \sigma\right)$ for $1 \leq i \leq e$ and one can compute a basis of any solution space in $(\mathbb{F}, \sigma)$, the above reduction technique immediately turns into an algorithm to compute a basis of the solution space $\left(\boldsymbol{a}, \boldsymbol{f}, \mathbb{F}_{e}\right)$. In particular our algorithm gives a reduction of $\left(\boldsymbol{a}, \boldsymbol{f}, \mathbb{F}_{e}\right)$ to $\mathbb{F}$ where we easily can collect the reduction tuple set of this reduction. Furthermore, if one stops collecting tuples in the subreductions of $\left(\boldsymbol{a}, \boldsymbol{f}, \mathbb{F}_{i}\right)$ to $\mathbb{F}$ when $\boldsymbol{a}$ is inhomogeneous, one can extract the $\mathbb{F}_{r}$-critical tuples in this reduction. Our algorithm can be found explicitly in [14].

Now assume that $(\mathbb{F}, \sigma)$ is a $\Pi \Sigma$-field over a $\sigma$-computable $\mathbb{K}$, i.e., $\left(\mathbb{F}\left(t_{1}\right) \ldots\left(t_{e}\right), \sigma\right)$ is a $\Pi \Sigma$-field over $\mathbb{K}$. Then by Theorem 4 there are algorithms to solve the denominator and polynomial degree bound problem. Moreover, for the special case $\mathbb{F}=\mathbb{K}$ there is the following

Base case II. If const $\sigma \mathbb{K}=\mathbb{K}, \mathbf{0} \neq \boldsymbol{a}=\left(a_{1}, a_{2}\right) \in \mathbb{K}^{2}$ and $\boldsymbol{f}=\left(f_{1}, \ldots, f_{n}\right) \in \mathbb{K}^{n}$ then $\mathrm{V}(\boldsymbol{a}, \boldsymbol{f}, \mathbb{K})=$ Nullspace $_{\mathbb{K}}\left(\boldsymbol{f}^{\prime}\right)$ for $\boldsymbol{f}^{\prime}=\left(f_{1}, \ldots, f_{n},-\left(a_{1}+a_{2}\right)\right)$. A basis can be computed by linear algebra; see [10].

Hence, with our algorithm one can compute a basis of $\mathrm{V}\left(\boldsymbol{a}, \boldsymbol{f}, \mathbb{F}\left(t_{1}\right) \ldots\left(t_{e}\right)\right)$ in a $\Pi \Sigma$-field $\left(\mathbb{F}\left(t_{1}\right) \ldots\left(t_{e}\right), \sigma\right)$ over a $\sigma$-computable $\mathbb{K}$ and can extract the $\mathbb{F}$-critical tuples of the corresponding reduction of $\left(\boldsymbol{a}, \boldsymbol{f}, \mathbb{F}\left(t_{1}\right) \ldots\left(t_{e}\right)\right)$ to $\mathbb{F}$. 
Finally, we introduce reductions to $\mathbb{F}$ that are extensionstable. Let $\left(\mathbb{F}\left(t_{1}\right) \ldots\left(t_{e}\right), \sigma\right)$ be a $\Pi \Sigma$-extension of $(\mathbb{F}, \sigma), \boldsymbol{a} \in$ $\left(\mathbb{H}\left[t_{e}\right]^{*}\right)^{2}$ and $\boldsymbol{f} \in \mathbb{H}\left[t_{e}\right]^{n}$ for $\mathbb{H}:=\mathbb{F}\left(t_{1}\right) \ldots\left(t_{e-1}\right)$. We call a denominator bound $d \in \mathbb{H}\left[t_{e}\right]^{*}$ of $\mathrm{V}\left(\boldsymbol{a}, \boldsymbol{f}, \mathbb{H}\left(t_{e}\right)\right)$ or a degree bound $b$ of $\mathrm{V}\left(\boldsymbol{a}, \boldsymbol{f}, \mathbb{H}\left[t_{e}\right]\right)$ extension-stable over $\mathbb{F}$ if $\boldsymbol{a}$ is inhomogeneous over $\mathbb{H}\left(t_{e}\right)$ or the following holds. Take any $\Sigma^{*}$-extension $\left(\mathbb{F}\left(t_{1}\right) \ldots\left(t_{e}\right)(s), \sigma\right)$ of $\left(\mathbb{F}\left(t_{1}\right) \ldots\left(t_{e}\right), \sigma\right)$ over $\mathbb{F}$, and embed $\boldsymbol{a}, \boldsymbol{f}$ in the reordered $\Pi \Sigma$-ext. $\left(\mathbb{F}(s)\left(t_{1}\right) \ldots\left(t_{e}\right), \sigma\right)$ of $(\mathbb{F}, \sigma)$. Then also $d$ embedded in $\mathbb{F}(s)\left(t_{1}\right) \ldots\left(t_{e}\right)$ must be a denominator bound of $\mathrm{V}\left(\boldsymbol{a}, \boldsymbol{f}, \mathbb{F}(s)\left(t_{1}\right) \ldots\left(t_{e}\right)\right)$. Similarly, $b$ must be a degree bound of $\mathrm{V}\left(\boldsymbol{a}, \boldsymbol{f}, \mathbb{F}(s)\left(t_{1}\right) \ldots\left(t_{e-1}\right)\left[t_{e}\right]\right)$. We call a reduction of $\mathrm{V}\left(\boldsymbol{a}, \boldsymbol{f}, \mathbb{F}\left(t_{1}\right) \ldots\left(t_{e}\right)\right)$ to $\mathbb{F}$ extensionstable if all denominator and degree bounds within the reduction to $\mathbb{F}$ are extension-stable over $\mathbb{F}$.

It has been shown in [10, Theorem 8.2] and [11, Theorem 7.3] that the algorithms proposed in [6] already compute extension-stable denominator and degree bounds in a $\Pi \Sigma$-field. Summarizing, we obtain

ThEOREM 5. Let $(\mathbb{E}, \sigma)$ be a $\Pi \Sigma$-field over a $\sigma$-computable $\mathbb{K}, \mathbf{0} \neq \boldsymbol{a} \in \mathbb{E}^{2}$ and $\boldsymbol{f} \in \mathbb{E}^{n}$. Then there is an algorithm that computes a basis of $\mathrm{V}(\boldsymbol{a}, \boldsymbol{f}, \mathbb{E})$ with an extension-stable reduction of $(\boldsymbol{a}, \boldsymbol{f}, \mathbb{E})$ to $\mathbb{F}$. Moreover, during this computation, one can extract the $\mathbb{F}$-critical tuples.

Example 3. In Example 2 the denominator and degree bounds are extension-stable. Consequently, this reduction of $\left((1,-1),\left(\sigma\left(t_{2} / t_{1}\right)\right), \mathbb{F}\left(t_{2}\right)\right)$ to $\mathbb{F}$ is extension-stable. The $\mathbb{F}$-critical tuples are $\left(\left(\left(t_{1}+1\right)^{2},-\left(t_{1}+1\right)^{2}\right), \boldsymbol{f}, \mathbb{F}\right)$ for $\boldsymbol{f} \in$ $\left\{(0),\left(t_{1}+1,-2\left(t_{1}+1\right)\right),\left(1,-\left(t_{1}+1\right)\right)\right\}$.

\section{REFINED SUMMATION ALGORITHMS}

In the sequel let $(\mathbb{E}, \sigma)$ with $\mathbb{E}:=\mathbb{F}\left(t_{1}\right) \ldots\left(t_{e}\right)$ be a $\Pi \Sigma$ field over a $\sigma$-computable $\mathbb{K}$ and $\boldsymbol{f} \in \mathbb{E}^{n}$. Then in Theorem 6 we will develop a constructive criterium which tells us if a $\Sigma^{*}$-extension of $(\mathbb{E}, \sigma)$ over $\mathbb{F}$ is $\mathbb{F}$-complete for $\boldsymbol{f}$ and how such an extension can be constructed. For this task we first compute a basis of $\mathbb{V}:=\mathrm{V}((1,-1), \boldsymbol{f}, \mathbb{E})$ together with an extension-stable reduction of $((1,-1), \boldsymbol{f}, \mathbb{E})$ to $\mathbb{F}$; see Theorem 5. If the dimension of $\mathbb{V}$ is $n+1$, the trivial extension $(\mathbb{E}, \sigma)$ of $(\mathbb{E}, \sigma)$ is clearly $\mathbb{F}$-complete for $\boldsymbol{f}$. Otherwise, we extract the $\mathbb{F}$-critical tuple set in our extension-stable reduction; see Theorem 5. Then the crucial observation is stated in Proposition 1 that depends on Lemma 3. This lemma is a special case of Karr's Fundamental Theorem [6, 7]; for a proof see [9, Proposition 4.1.2].

Lemma 3. If $(\mathbb{E}, \sigma)$ is a $\Sigma^{*}$-extension of $(\mathbb{F}, \sigma), \mathbf{0} \neq \boldsymbol{a} \in$ $\mathbb{F}^{2}$ inhomogeneous over $\mathbb{F}$ and $\boldsymbol{f} \in \mathbb{F}^{n}$ then $\mathrm{V}(\boldsymbol{a}, \boldsymbol{f}, \mathbb{E})=$ $\mathrm{V}(\boldsymbol{a}, \boldsymbol{f}, \mathbb{F})$.

Proposition 1. Let $(\mathbb{E}(s), \sigma)$ with $\mathbb{E}:=\mathbb{F}\left(t_{1}\right) \ldots\left(t_{e}\right)$ be a $\Pi \Sigma$-extension of $(\mathbb{F}, \sigma)$ with $\sigma(s)-s \in \mathbb{F}$ and consider the reordered $\Pi \Sigma$-extension $\left(\mathbb{F}(s)\left(t_{1}\right) \ldots\left(t_{e}\right), \sigma\right)$ of $(\mathbb{F}, \sigma)$. Let $\boldsymbol{a} \in$ $\mathbb{E}^{2}$ be homogeneous over $\mathbb{E}, \boldsymbol{f} \in \mathbb{E}^{n}$, and let $S$ be an $\mathbb{F}$-critical tuple set of an extension-stable reduction of $(\boldsymbol{a}, \boldsymbol{f}, \mathbb{E})$ to $\mathbb{F}$. If for all $\left(\boldsymbol{a}^{\prime}, \boldsymbol{f}^{\prime}, \mathbb{F}\right) \in S$ we have $\mathrm{V}\left(\boldsymbol{a}^{\prime}, \boldsymbol{f}^{\prime}, \mathbb{F}\right)=\mathrm{V}\left(\boldsymbol{a}^{\prime}, \boldsymbol{f}^{\prime}, \mathbb{F}(s)\right)$ then $\mathrm{V}(\boldsymbol{a}, \boldsymbol{f}, \mathbb{E})=\mathrm{V}(\boldsymbol{a}, \boldsymbol{f}, \mathbb{E}(s))=\mathrm{V}\left(\boldsymbol{a}, \boldsymbol{f}, \mathbb{F}(s)\left(t_{1}\right) \ldots\left(t_{e}\right)\right)$.

Proof. The proof will be done by induction on the number $e$ of extensions $\mathbb{F}\left(t_{1}\right) \ldots\left(t_{e}\right)$. First consider the case $e=0$. Since $\boldsymbol{a}$ is homogeneous, $(\boldsymbol{a}, \boldsymbol{f}, \mathbb{F}) \in S$ and therefore $\mathrm{V}(\boldsymbol{a}, \boldsymbol{f}, \mathbb{F}(s))=\mathrm{V}(\boldsymbol{a}, \boldsymbol{f}, \mathbb{F})$. Now assume that the proposition holds for $e \geq 0$. Let $\left(\mathbb{F}\left(t_{1}\right) \ldots\left(t_{e}\right)\left(t_{e+1}\right)(s), \sigma\right)$ be a
$\Pi \Sigma$-extension of $(\mathbb{F}, \sigma)$ with $\sigma(s)-s \in \mathbb{F}$ and consider the reordered $\Pi \Sigma$-extension $\left(\mathbb{F}(s)\left(t_{1}\right) \ldots\left(t_{e}\right)\left(t_{e+1}\right), \sigma\right)$ of $(\mathbb{F}, \sigma)$. As shortcut write $\mathbb{E}:=\mathbb{F}\left(t_{1}\right) \ldots\left(t_{e}\right), \mathbb{H}:=\mathbb{F}(s)\left(t_{1}\right) \ldots\left(t_{e}\right)$. Let $\boldsymbol{f} \in \mathbb{E}\left(t_{e+1}\right)^{n}$, assume that $\boldsymbol{a} \in \mathbb{E}\left(t_{e+1}\right)^{2}$ is homogeneous over $\mathbb{E}\left(t_{e+1}\right)$, and take any extension-stable reduction of $\left(\boldsymbol{a}, \boldsymbol{f}, \mathbb{E}\left(t_{e+1}\right)\right)$ to $\mathbb{F}$ with the $\mathbb{F}$-critical tuple set $S$. Now suppose that $\mathrm{V}\left(\boldsymbol{a}^{\prime}, \boldsymbol{f}^{\prime}, \mathbb{F}\right)=\mathrm{V}\left(\boldsymbol{a}^{\prime}, \boldsymbol{f}^{\prime}, \mathbb{F}(s)\right)$ for all $\left(\boldsymbol{a}^{\prime}, \boldsymbol{f}^{\prime}, \mathbb{F}\right) \in$ $S$. Then we will show that

$$
\mathrm{V}\left(\boldsymbol{a}, \boldsymbol{f}, \mathbb{E}\left(t_{e+1}\right)\right)=\mathrm{V}\left(\boldsymbol{a}, \boldsymbol{f}, \mathbb{H}\left(t_{e+1}\right)\right) .
$$

In the extension-stable reduction let $d \in \mathbb{E}\left[t_{e+1}\right]^{*}$ be the denominator bound of $\mathrm{V}\left(\boldsymbol{a}, \boldsymbol{f}, \mathbb{E}\left(t_{e+1}\right)\right)$. Since $\boldsymbol{a}$ is homogeneous over $\mathbb{E}\left(t_{e+1}\right), d \in \mathbb{H}\left[t_{e+1}\right]$ is also a denominator bound of $\mathrm{V}\left(\boldsymbol{a}, \boldsymbol{f}, \mathbb{H}\left(t_{e+1}\right)\right)$. After clearing denominators and cancelling common factors, we get $\boldsymbol{a}^{\prime}:=\left(a_{1} / \sigma(d), a_{2} / d\right) q \in$ $\mathbb{E}\left[t_{e+1}\right]^{2}$ and $\boldsymbol{f}^{\prime}:=\boldsymbol{f} q \in \mathbb{E}\left[t_{e+1}\right]^{n}$ for some $q \in \mathbb{E}\left(t_{e+1}\right)^{*}$ in our reduction. Note that $\boldsymbol{a}^{\prime}$ is still homogeneous over $\mathbb{E}\left(t_{e+1}\right)$. This follows from the fact that if for $h \in \mathbb{E}\left(t_{e+1}\right)$ we have $a_{1} \sigma(h)+a_{2} h=0$ then $a_{1}^{\prime} \sigma(h d)+a_{2}^{\prime} h d=0$. Now it suffices to show that $\mathrm{V}\left(\boldsymbol{a}^{\prime}, \boldsymbol{f}^{\prime}, \mathbb{H}\left[t_{e+1}\right]\right)=\mathrm{V}\left(\boldsymbol{a}^{\prime}, \boldsymbol{f}^{\prime}, \mathbb{E}\left[t_{e+1}\right]\right)$, in order to show (4). In the given reduction let $b$ be the degree bound of $\mathrm{V}\left(\boldsymbol{a}^{\prime}, \boldsymbol{f}^{\prime}, \mathbb{E}\left[t_{e+1}\right]\right)$. Since $\boldsymbol{a}^{\prime}$ is homogeneous over $\mathbb{E}\left(t_{e+1}\right), b$ is a degree bound of $\mathrm{V}\left(\boldsymbol{a}^{\prime}, \boldsymbol{f}^{\prime}, \mathbb{H}\left[t_{e+1}\right]\right)$ too. Hence, if $\mathrm{V}\left(\boldsymbol{a}^{\prime}, \boldsymbol{f}^{\prime}, \mathbb{E}\left[t_{e+1}\right]_{b}\right)=\mathrm{V}\left(\boldsymbol{a}^{\prime}, \boldsymbol{f}^{\prime}, \mathbb{H}\left[t_{e+1}\right]_{b}\right)$, also (4) is proven. Let $\left(\left(\boldsymbol{a}, \boldsymbol{f}_{\boldsymbol{b}}, \mathbb{E}\left[t_{e+1}\right]_{b}\right), \ldots,\left(\boldsymbol{a}, \boldsymbol{f}_{-\mathbf{1}}, \mathbb{E}\left[t_{e+1}\right]_{-1}\right)\right)$ be the incremental tuples and $\left(\left(\tilde{\boldsymbol{a}}_{\boldsymbol{b}}, \tilde{\boldsymbol{f}}_{\boldsymbol{b}}, \mathbb{E}\right), \ldots,\left(\tilde{\boldsymbol{a}}_{\mathbf{0}}, \tilde{\boldsymbol{f}}_{\mathbf{0}}, \mathbb{E}\right)\right)$ be the coefficient-tuples in the incr. reduction of $\left(\boldsymbol{a}, \boldsymbol{f}, \mathbb{E}\left[t_{e+1}\right]_{b}\right)$. We show that $\mathrm{V}\left(\tilde{\boldsymbol{a}}_{\boldsymbol{i}}, \tilde{\boldsymbol{f}}_{\boldsymbol{i}}, \mathbb{E}\right)=\mathrm{V}\left(\tilde{\boldsymbol{a}}_{\boldsymbol{i}}, \tilde{\boldsymbol{f}}_{\boldsymbol{i}}, \mathbb{H}\right)$ for all $0 \leq i \leq$ $b$. By reordering of $\left(\mathbb{F}\left(t_{1}\right) \ldots\left(t_{e+1}\right)(s), \sigma\right)$ we get the $\Pi \Sigma$ extension $\left(\mathbb{F}\left(t_{1}\right) \ldots\left(t_{e}\right)(s)\left(t_{e+1}\right), \sigma\right)$ of $(\mathbb{F}, \sigma)$. First suppose that $\tilde{\boldsymbol{a}}_{\boldsymbol{i}}$ is inhomogeneous over $\mathbb{E}$. Hence, $\mathrm{V}\left(\tilde{\boldsymbol{a}}_{\boldsymbol{i}}, \tilde{\boldsymbol{f}}_{\boldsymbol{i}}, \mathbb{E}\right)=$ $\mathrm{V}\left(\tilde{\boldsymbol{a}}_{\boldsymbol{i}}, \tilde{\boldsymbol{f}}_{\boldsymbol{i}}, \mathbb{E}(s)\right)$ by Lemma 3 , and therefore $\mathrm{V}\left(\tilde{\boldsymbol{a}}_{\boldsymbol{i}}, \tilde{\boldsymbol{f}}_{\boldsymbol{i}}, \mathbb{E}\right)=$ $\mathrm{V}\left(\tilde{\boldsymbol{a}}_{\boldsymbol{i}}, \tilde{\boldsymbol{f}}_{\boldsymbol{i}}, \mathbb{H}\right)$ by $\left(\mathbb{F}\left(t_{1}\right) \ldots\left(t_{e}\right)(s), \sigma\right) \simeq\left(\mathbb{F}(s)\left(t_{1}\right) \ldots\left(t_{e}\right), \sigma\right)$. Otherwise, assume that $\tilde{\boldsymbol{a}}_{\boldsymbol{i}}$ is homogeneous over $\mathbb{E}$. Then the extension-stable reduction of $\left(\boldsymbol{a}, \boldsymbol{f}, \mathbb{E}\left(t_{e+1}\right)\right)$ to $\mathbb{F}$ contains an extension-stable reduction of $\left(\tilde{\boldsymbol{a}}_{\boldsymbol{i}}, \tilde{\boldsymbol{f}}_{\boldsymbol{i}}, \mathbb{E}\right)$ to $\mathbb{F}$ and the $\mathbb{F}$ critical tuple set of the reduction of $\left(\tilde{\boldsymbol{a}}_{\boldsymbol{i}}, \tilde{\boldsymbol{f}}_{\boldsymbol{i}}, \mathbb{E}\right)$ is a subset of $S$. Hence with the induction assumption it follows that $\mathrm{V}\left(\tilde{\boldsymbol{a}}_{\boldsymbol{i}}, \tilde{\boldsymbol{f}}_{\boldsymbol{i}}, \mathbb{E}\right)=\mathrm{V}\left(\tilde{\boldsymbol{a}}_{\boldsymbol{i}}, \tilde{\boldsymbol{f}}_{\boldsymbol{i}}, \mathbb{H}\right)$. Since $\mathbb{E}\left[t_{e+1}\right]_{-1}=\mathbb{H}\left[t_{e+1}\right]_{-1}=$ $\{0\}$, we have $\mathrm{V}\left(\boldsymbol{a}, \boldsymbol{f}_{-\mathbf{1}}, \mathbb{E}\left[t_{e+1}\right]_{-1}\right)=\mathrm{V}\left(\boldsymbol{a}, \boldsymbol{f}_{-\mathbf{1}}, \mathbb{H}\left[t_{e+1}\right]_{-1}\right)$. Consequently by the construction of the incremental reduction, see [14], we can conclude that $\mathrm{V}\left(\boldsymbol{a}, \boldsymbol{f}_{\boldsymbol{i}}, \mathbb{E}\left[t_{e+1}\right]_{i}\right)=$ $\mathrm{V}\left(\boldsymbol{a}, \boldsymbol{f}_{\boldsymbol{i}}, \mathbb{H}\left[t_{e+1}\right]_{i}\right)$ for all $-1 \leq i \leq b$ and therefore we have proven (4). Hence $\mathrm{V}\left(\boldsymbol{a}, \boldsymbol{f}, \mathbb{E}\left(t_{e+1}\right)\right)=\mathrm{V}\left(\boldsymbol{a}, \boldsymbol{f}, \mathbb{E}\left(t_{e+1}\right)(s)\right)$ by reordering of $\mathbb{F}(s)\left(t_{1}\right) \ldots\left(t_{e+1}\right)$ to $\mathbb{F}\left(t_{1}\right) \ldots\left(t_{e+1}\right)(s)$.

Hence we have $\mathrm{V}((1,-1), \boldsymbol{f}, \mathbb{E}) \subsetneq \mathrm{V}((1,-1), \boldsymbol{f}, \mathbb{E}(s))$ for a $\Sigma^{*}$-extension $(\mathbb{E}(s), \sigma)$ of $(\mathbb{E}, \sigma)$ over $\mathbb{F}$ if $\mathrm{V}\left(\boldsymbol{a}^{\prime}, \boldsymbol{f}^{\prime}, \mathbb{F}\right) \subsetneq$ $\mathrm{V}\left(\boldsymbol{a}^{\prime}, \boldsymbol{f}^{\prime}, \mathbb{F}(s)\right)$ in one of its $\mathbb{F}$-critical tuples $\left(\boldsymbol{a}^{\prime}, \boldsymbol{f}^{\prime}, \mathbb{F}\right)$ in an extension-stable reduction to $\mathbb{F}$.

Example 4. Consider the $\Pi \Sigma$-fields from Example 1, 2 and 3. By Example 1 it follows that $\mathrm{V}\left((1,-1),\left(\frac{\sigma\left(t_{2}\right)}{t_{1}}\right), \mathbb{F}\left(t_{2}\right)\right)$ is a proper subset of $\mathrm{V}\left((1,-1),\left(\frac{\sigma\left(t_{2}\right)}{t_{1}}\right), \mathbb{F}\left(t_{2}\right)(s)\right)$. Hence looking at the $\mathbb{F}$-critical tuples of our extension stable reduction in Example 3, we know by Proposition 1 that there is an $\boldsymbol{f} \in\left\{(0),\left(t_{1}+1,-2\left(t_{1}+1\right)\right),\left(1,-\left(t_{1}+1\right)\right)\right\}$ such that $\mathrm{V}(\boldsymbol{a}, \boldsymbol{f}, \mathbb{F})$ with $\boldsymbol{a}=\left(\left(t_{1}+1\right)^{2},-\left(t_{1}+1\right)^{2}\right)$ is a proper subset of $\mathrm{V}(\boldsymbol{a}, \boldsymbol{f}, \mathbb{F}(s))$. Indeed, we can choose $\boldsymbol{f}=\left(1,-\left(t_{1}+1\right)\right)$ since there does not exist a $g \in \mathbb{F}$ with $\sigma(g)-g=\frac{1}{\left(t_{1}+1\right)^{2}}$, but there is the solution $g=s \in \mathbb{F}(s)$. 
Next we provide a sufficient condition in Proposition 2 which tells us if a $\Sigma^{*}$-extension cannot contribute further to a given solution space.

Proposition 2. Let $(\mathbb{F}, \sigma)$ be a difference field with $\boldsymbol{a}=$ $\left(a_{1}, a_{2}\right) \in \mathbb{F}^{2}$ homogeneous over $\mathbb{F}$ and $\boldsymbol{f}=\left(f_{1}, \ldots, f_{n}\right) \in$ $\mathbb{F}^{n}$. If for all $1 \leq i \leq n$ there is a $g \in \mathbb{F}^{*}$ with $a_{1} \sigma(g)+$ $a_{2} g=f_{i}$ then for any difference field (ring) extension $(\mathbb{E}, \sigma)$ of $(\mathbb{F}, \sigma)$ with const $_{\sigma} \mathbb{E}=$ const $_{\sigma} \mathbb{F}$ we have $\mathrm{V}(\boldsymbol{a}, \boldsymbol{f}, \mathbb{F})=$ $\mathrm{V}(\boldsymbol{a}, \boldsymbol{f}, \mathbb{E})$.

Proof. Let $g_{i} \in \mathbb{F}$ with $a_{1} \sigma\left(g_{0}\right)+a_{2} g_{0}=0$ and $a_{1} \sigma\left(g_{i}\right)+$ $a_{2} g_{i}=f_{i}$ for $1 \leq i \leq n$. Then observe that $\left(0, \ldots, 0, g_{0}\right)$, $\left(1,0, \ldots, 0, g_{1}\right), \ldots,\left(0, \ldots, 0,1, g_{n}\right) \in \mathbb{K}^{n} \times \mathbb{F}$ forms a basis of $\mathbb{V}:=\mathrm{V}(\boldsymbol{a}, \boldsymbol{f}, \mathbb{F})$ over $\mathbb{K}:=$ const $_{\sigma} \mathbb{F}$. Since $\mathbb{V}$ is a subspace of $\mathbb{W}:=\mathrm{V}(\boldsymbol{a}, \boldsymbol{f}, \mathbb{E})$ over $\mathbb{K}$ and the dimension of $\mathbb{W}$ is at most $n+1$, it follows that $\mathbb{V}=\mathbb{W}$.

This result allows us to specify a criterium in Theorem 6 if a $\Sigma^{*}$-extension of $(\mathbb{E}, \sigma)$ over $\mathbb{F}$ is $\mathbb{F}$-complete for $\boldsymbol{f}$.

Theorem 6. Let $(\mathbb{E}, \sigma)$ with $\mathbb{E}:=\mathbb{F}\left(t_{1}\right) \ldots\left(t_{e}\right)$ be a $\Pi \Sigma$ extension of $(\mathbb{F}, \sigma)$ and $\boldsymbol{f} \in \mathbb{E}^{n}$. Let $\left\{\left(\boldsymbol{a}_{\boldsymbol{i}}, \boldsymbol{f}_{\boldsymbol{i}}, \mathbb{F}\right)\right\}_{1 \leq i \leq k}$ with $\boldsymbol{a}_{\boldsymbol{i}}=\left(a_{i 1}, a_{i 2}\right)$ and $\boldsymbol{f}_{\boldsymbol{i}}=\left(f_{i 1}, \ldots, f_{i r_{i}}\right) \in \mathbb{F}^{r_{i}}$ be the $\mathbb{F}$-critical tuple set of an extension-stable reduction of $\mathrm{V}((1,-1), \boldsymbol{f}, \mathbb{E})$ to $\mathbb{F}$. If $(\mathbb{G}, \sigma)$ is a $\Sigma^{*}$-extension of $(\mathbb{E}, \sigma)$ over $\mathbb{F}$ where for any $1 \leq i \leq k$ and $1 \leq j \leq r_{i}$ there is a $g \in \mathbb{G}^{*}$ with $a_{i 1} \sigma(g)-a_{i 2} g=f_{i j}$ then the extension is $\mathbb{F}$-complete for $\boldsymbol{f}$.

Proof. Suppose such an extension $(\mathbb{G}, \sigma)$ of $(\mathbb{E}, \sigma)$ over $\mathbb{F}$ is not $\mathbb{F}$-complete for $\boldsymbol{f}$. Then we can take a $c \in \mathbb{K}^{n}$ such that $\sigma(g)-g=\boldsymbol{c} \boldsymbol{f}$ has a solution in some $\Pi \Sigma$-extension of $(\mathbb{E}, \sigma)$, but no solution in $(\mathbb{G}, \sigma)$ and therefore no solution in $(\mathbb{E}, \sigma)$. Hence, by Lemma 1 there is a $\Sigma^{*}$-extension $(\mathbb{E}(s), \sigma)$ of $(\mathbb{E}, \sigma)$ over $\mathbb{F}$ and a $g \in \mathbb{E}(s)$ with $\sigma(g)-g=\boldsymbol{c} \boldsymbol{f}$. Consequently, by Proposition 1 there exists an $i$ with $1 \leq i \leq k$ such that $\mathrm{V}\left(\boldsymbol{a}_{\boldsymbol{i}}, \boldsymbol{f}_{\boldsymbol{i}}, \mathbb{F}\right) \subsetneq \mathrm{V}\left(\boldsymbol{a}_{\boldsymbol{i}}, \boldsymbol{f}_{\boldsymbol{i}}, \mathbb{F}(s)\right)$ holds for the $\Sigma^{*}$ extension $(\mathbb{F}(s), \sigma)$ of $(\mathbb{F}, \sigma)$. But by Proposition 2 we have $\mathrm{V}\left(\boldsymbol{a}_{\boldsymbol{i}}, \boldsymbol{f}_{\boldsymbol{i}}, \mathbb{F}\right)=\mathrm{V}\left(\boldsymbol{a}_{\boldsymbol{i}}, \boldsymbol{f}_{\boldsymbol{i}}, \mathbb{F}(s)\right)$, a contradiction.

Example 5. Consider Examples 2 and 3. Since for any $f \in$ $\left\{0, t_{1}+1,-2\left(t_{1}+1\right), 1,-\left(t_{1}+1\right)\right\}$ there is a $g \in \mathbb{F}\left(t_{2}\right)(s)$ with $\sigma(g)-g=f$, it follows that the $\Sigma^{*}$-extension $\left(\mathbb{F}\left(t_{2}\right)(s), \sigma\right)$ of $\left(\mathbb{F}\left(t_{2}\right), \sigma\right)$ is $\mathbb{F}$-complete for $\left(\sigma\left(t_{2}\right) / t_{1}\right)$.

Finally, in Proposition 3 we show that such an extension can be constructed that fulfills our sufficient criterium.

Proposition 3. Let $(\mathbb{E}, \sigma)$ be a $\Pi \Sigma$-extension of $(\mathbb{F}, \sigma)$, $\left(a_{i 1}, a_{i 2}\right) \in \mathbb{F}^{2}$ be homogeneous over $\mathbb{F}$ and $f_{i} \in \mathbb{F}$ for $1 \leq$ $i \leq n$. Then there is a $\Sigma^{*}$-extension $(\mathbb{G}, \sigma)$ of $(\mathbb{E}, \sigma)$ over $\mathbb{F}$ such that there is a $g \in \mathbb{G}^{*}$ with $a_{i 1} \sigma(g)+a_{i 2} g=f_{i}$ for all $1 \leq i \leq n$. If $(\mathbb{F}, \sigma)$ is a $\Pi \Sigma$-field over a $\sigma$-computable $\mathbb{K}$, such a $\Pi \Sigma$-field $(\mathbb{G}, \sigma)$ can be computed.

Proof. Suppose that we have shown the existence for such a $\Sigma^{*}$-extension $(\mathbb{G}, \sigma)$ of $(\mathbb{E}, \sigma)$ over $\mathbb{F}$ for $1 \leq i \leq n$. Now let $\left(a_{1}, a_{2}\right) \in \mathbb{F}^{2}$ be homogeneous over $\mathbb{F}$ and $f \in$ $\mathbb{F}$. If there is a $g \in \mathbb{G}$ with $a_{1} \sigma(g)+a_{2} g=f$, we have shown the induction step. Otherwise, construct the extension $(\mathbb{G}(s), \sigma)$ of $(\mathbb{G}, \sigma)$ with $s$ transcendental over $\mathbb{F}$ and $\sigma(s)=s-\frac{f}{h a_{2}} \in \mathbb{F}$ where $h \in \mathbb{F}^{*}$ with $a_{1} \sigma(h)+a_{2} h=0$. Now suppose there is a $g^{\prime} \in \mathbb{G}^{*}$ with $\sigma\left(g^{\prime}\right)-g^{\prime}=-\frac{f}{h a_{2}}$. Then for $w:=h g^{\prime} \in \mathbb{G}^{*}$ we have $f=-a_{2} h\left(\sigma\left(g^{\prime}\right)-g^{\prime}\right)=$ $a_{1} \sigma(h) \sigma\left(g^{\prime}\right)+a_{2} h g^{\prime}=a_{1} \sigma(w)+a_{2} w$, a contradiction.
Hence by Theorem $1(\mathbb{G}(s), \sigma)$ is a $\Sigma^{*}$-extension of $(\mathbb{G}, \sigma)$ over $\mathbb{F}$. Furthermore, for $v:=h s \in \mathbb{G}(s)$ we have that $a_{1} \sigma(v)+a_{2} v=f$, which follows by similar arguments as above for $w$. This closes the induction step.

Now suppose that $(\mathbb{F}, \sigma)$ is a $\Pi \Sigma$-field over a $\sigma$-computable $\mathbb{K}$. Then by Theorem 5 one can decide if there exists a $g \in \mathbb{G}^{*}$ with $a_{1} \sigma(g)+a_{2} g=f$ and can compute an $h \in \mathbb{F}^{*}$ with $a_{1} \sigma(h)+a_{2} h=0$. This shows, that the proof above becomes completely constructive.

Summarizing, we first compute a basis of $\mathrm{V}((1,-1), \boldsymbol{f}, \mathbb{E})$ with an extension-stable reduction and extract the $\mathbb{F}$-critical tuples; this is possible by Theorem 5 . Next we construct with Proposition 3 a $\Sigma^{*}$-extension of $(\mathbb{E}, \sigma)$ over $\mathbb{F}$ that fulfills the criterium in Theorem 6 .

Example 6. Looking at Example 3 we obtain immediately the $\Sigma^{*}$-extension $\left(\mathbb{F}\left(t_{2}\right)(s), \sigma\right)$ of $\left(\mathbb{F}\left(t_{2}\right), \sigma\right)$ with $\sigma(s)=s+$ $\frac{1}{\left(t_{1}+1\right)^{2}}$ which is $\mathbb{F}$-complete for $\left(\sigma\left(t_{2} / t_{1}\right)\right) \in \mathbb{F}\left(t_{2}\right)^{1}$ by following this strategy. Finally we restart our computation in this extension and obtain for $\mathrm{V}\left((1,-1),\left(\sigma\left(t_{2} / t_{1}\right)\right), \mathbb{F}\left(t_{2}\right)(s)\right)$ the basis $\left\{(0,1),\left(2, t_{2}+s\right)\right\}$ which gives the result $g=\frac{t_{2}+s}{2}$ in Example 1.

Now we proceed, and try to find a $g^{\prime} \in \mathbb{F}\left(t_{2}\right)(s)$ such that $\sigma\left(g^{\prime}\right)-g^{\prime}=\sigma\left(g / t_{1}\right)$, but we fail. Therefore, we extract the $\mathbb{F}$-critical tuples $\left(\left(\left(t_{1}+1\right)^{3},-\left(t_{1}+1\right)^{3}\right), \boldsymbol{f}, \mathbb{F}\right)$ with

$$
\begin{aligned}
& f \in\left\{\left(-\left(t_{1}+1\right)^{2}, \frac{t_{1}+1}{2},-2\left(t_{1}+1\right)\right),\left(2\left(t_{1}+1\right)^{2}, 0,0\right),\right. \\
& \left.(0,0),\left(-3\left(t_{1}+1\right)^{2},\left(t_{1}+1\right)^{2}, 0\right),\left(\left(t_{1}+1\right), 2,\left(t_{1}+1\right)^{2}\right)\right\}
\end{aligned}
$$

from our extension stable reduction to $\mathbb{F}$. Following Theorem 6 we construct a $\Sigma^{*}$-extension $(\mathbb{G}, \sigma)$ of $\left(\mathbb{F}\left(t_{2}\right)(s), \sigma\right)$ over $\mathbb{F}$ such that there are $h \in \mathbb{G}$ with $\sigma(h)-h=\frac{f}{\left(t_{1}+1\right)^{2}}$ for all $f \in \mathbb{F}$ from (5). Following the algorithm given in the proof of Proposition 3 we obtain the $\Sigma^{*}$-extension $\left(\mathbb{F}\left(t_{2}\right)(s)\left(s^{\prime}\right), \sigma\right)$ of $\left(\mathbb{F}\left(t_{2}\right)(s), \sigma\right)$ with $\sigma\left(s^{\prime}\right)=s^{\prime}+\frac{2}{\left(t_{1}+1\right)^{3}}$; afterwards we cancel the constant factor 2. By Theorem 6 this extension is $\mathbb{F}$-complete for $\left(\sigma\left(g / t_{1}\right)\right) \in \mathbb{F}\left(t_{2}\right)(s)^{1}$. To this end we compute for the solution space $\mathrm{V}\left((1,-1),\left(\sigma\left(g / t_{1}\right)\right), \mathbb{F}\left(t_{2}\right)(s)\left(s^{\prime}\right)\right)$ the basis $\left\{(0,1),\left(6,\left(t_{2}^{3}+3 t_{2} s+2 s^{\prime}\right)\right)\right\}$ which gives the final result in Example 1.

Let $I \subseteq\{0, \ldots, e\}$. Restricting Algorithm 1 to $I=\{0\}$ gives just the above strategy.

In addition, $\mathbb{F}_{i}:=\mathbb{F}\left(t_{1}\right) \ldots\left(t_{i}\right)$-complete extensions can be searched for all $i \in I$. This can be motivated as follows. $\mathbb{F}_{i^{-}}$ complete extensions $\left(\mathbb{E}_{i}, \sigma\right)$ of $(\mathbb{E}, \sigma)$ with bigger $i$ can give more solutions $\mathbb{W}_{i}:=\Pi_{n}\left(\mathrm{~V}\left((1,-1), \boldsymbol{f}, \mathbb{E}_{i}\right)\right.$; but they might be also more complicated, since they depend on more $t_{j}$ (which are usually more nested). Hence, one should look for extensions with smallest possible $i$ that give still interesting solutions in $\mathbb{W}_{i}$. Algorithm 1 enables one to search in one stroke for all those $\mathbb{F}_{i}$-complete extensions with $i \in I$.

\section{Algorithm 1. SingleNestedCompleteExt $\left(\left(\mathbb{E}_{0}, \sigma\right), \boldsymbol{f}\right)$}

Input: A $\Pi \Sigma$-field $\left(\mathbb{E}_{0}, \sigma\right)$ with $\mathbb{E}_{0}:=\mathbb{F}\left(t_{1}\right) \ldots\left(t_{e}\right)$ over a $\sigma$-computable $\mathbb{K}, I=\left\{j_{1}<\cdots<j_{\lambda}\right\} \subseteq\{0, \ldots, e\}$ and $f \in \mathbb{E}_{0}^{n}$.

Output: $\Sigma^{*}$-extensions $\left(\mathbb{E}_{i}, \sigma\right)$ of $\left(\mathbb{E}_{i-1}, \sigma\right)$ over $\mathbb{F}\left(t_{1}\right) \ldots\left(t_{j_{i}}\right)$ which are single-nested $\mathbb{F}\left(t_{1}\right) \ldots\left(t_{j_{i}}\right)$-complete for $\boldsymbol{f}$ for $1 \leq$ $i \leq \lambda$; a basis of $\mathrm{V}\left((1,-1), \boldsymbol{f}, \mathbb{E}_{\lambda}\right)$. 
(1) Compute a basis $B$ of $\mathrm{V}\left((1,-1), \boldsymbol{f}, \mathbb{E}_{0}\right)$ by an extensionstable reduction to $\mathbb{F}$. Let $d:=\operatorname{dim} \mathrm{V}\left((1,-1), \boldsymbol{f}, \mathbb{E}_{0}\right)$.

(2) IF $d=n+1$ RETURN $\left(\left(\mathbb{E}_{0}, \sigma\right), B\right)$ FI

(3) FOR $i=1$ TO $\lambda$ DO

Extract the $\mathbb{F}\left(t_{1}\right) \ldots\left(t_{j_{\lambda}}\right)$-critical tuples from our reduction, say $\left\{\left(\boldsymbol{a}_{\boldsymbol{i}}, \boldsymbol{f}_{\boldsymbol{i}}, \mathbb{F}\right)\right\}_{1 \leq i \leq k}$, where $\boldsymbol{a}_{\boldsymbol{i}}=\left(a_{i 1}, a_{i 2}\right)$ and $\boldsymbol{f}_{\boldsymbol{i}}=\left(f_{i 1}, \ldots, f_{i r_{i}}\right) \in \mathbb{F}^{r_{i}}$ with $r_{i}>0$. Construct a singlenested $\Sigma^{*}$-ext. $\left(\mathbb{E}_{i}, \sigma\right)$ of $\left(\mathbb{E}_{i-1}, \sigma\right)$ over $\mathbb{F}\left(t_{1}\right) \ldots\left(t_{j_{\lambda}}\right)$ s.t. for any $1 \leq i \leq k$ and $1 \leq j \leq r_{i}$ there is a $g \in \mathbb{E}_{i}^{*}$ with $a_{i 1} \sigma(g)-a_{i 2} g=f_{i j}$.

OD

(4) IF $\left(\mathbb{E}_{\lambda}, \sigma\right)=\left(\mathbb{E}_{0}, \sigma\right) \operatorname{RETURN}\left(\left(\mathbb{E}_{0}, \sigma\right), B\right) \mathrm{FI}$

(5) Compute a basis $B^{\prime}$ of $\mathrm{V}\left((1,-1), \boldsymbol{f}, \mathbb{E}_{\lambda}\right)$ with dim. $d^{\prime}$.

(6) IF $d=d^{\prime}$ THEN $\operatorname{RETURN}\left(\left(\mathbb{E}_{0}, \sigma\right), B\right) \operatorname{ELSE} \operatorname{RETURN}\left(\left(\mathbb{E}_{\lambda}, \sigma\right), B^{\prime}\right)$. FI

TheOREM 7. Let $\left(\mathbb{E}_{0}, \sigma\right)$ with $\mathbb{E}_{0}:=\mathbb{F}\left(t_{1}\right) \ldots\left(t_{e}\right)$ be a $\Pi \Sigma$-field over a $\sigma$-computable $\mathbb{K}, I=\left\{j_{1}<\cdots<j_{\lambda}\right\} \subseteq$ $\{0, \ldots, e\}$ and $\boldsymbol{f} \in \mathbb{E}_{0}^{n}$. Then with Algorithm $1 \Sigma^{*}$-extensions $\left(\mathbb{E}_{i}, \sigma\right)$ of $\left(\mathbb{E}_{i-1}, \sigma\right)$ over $\mathbb{F}\left(t_{1}\right) \ldots\left(t_{j_{i}}\right)$ can be computed which are $\mathbb{F}\left(t_{1}\right) \ldots\left(t_{j_{i}}\right)$-complete for $\boldsymbol{f}$ for $1 \leq i \leq \lambda$.

The $\Sigma^{*}$-extension $\left(\mathbb{E}_{\lambda}, \sigma\right)$ of $(\mathbb{E}, \sigma)$ over $\mathbb{F}$ produced by Algorithm 1 can be reduced to a more compact extension that delivers the same solutions $\Pi_{n}\left(\mathrm{~V}\left((1,-1), \boldsymbol{f}, \mathbb{E}_{\lambda}\right)\right)$. Namely, if $\mathbb{E}_{\lambda}:=\mathbb{E}\left(s_{1}\right) \ldots\left(s_{\epsilon}\right)$, remove those $s_{i}$ that do not occur in $\mathbb{W}_{\lambda}=\mathrm{V}\left((1,-1), \boldsymbol{f}, \mathbb{E}_{\lambda}\right)$. Moreover, join all those $s_{i}$ 's to one single $\Sigma^{*}$-extension which occur in a basis element of $\mathbb{W}_{i}$; see Lemma 1. Furthermore, cancel constants from $\mathbb{K}$ that may occur in the summand $\sigma\left(s_{i}\right)-s_{i}$; see Example 6 .

Observe that recursively applied indefinite summation can be treated more efficiently, if one reduces these extensions after each application of Algorithm 1.

\section{Acknowledgments}

I would like to thank the referees for their valuable comments and suggestions.

\section{REFERENCES}

[1] S. A. Abramov and M. Petkovšek. D'Alembertian solutions of linear differential and difference equations. In J. von zur Gathen, editor, Proc. ISSAC'94, pages 169-174. ACM Press, Baltimore, 1994.

[2] M. Bronstein. On solutions of linear ordinary difference equations in their coefficient field. J. Symbolic Comput., 29(6):841-877, June 2000.
[3] R. M. Cohn. Difference Algebra. Interscience Publishers, John Wiley \& Sons, 1965.

[4] K. Driver, H. Prodinger, C. Schneider, and A. Weideman. Padé approximations to the logarithm III: Alternative methods and additional results. To Appear in Ramanujan Journal, 2004.

[5] P. A. Hendriks and M. F. Singer. Solving difference equations in finite terms. J. Symbolic Comput., 27(3):239-259, 1999.

[6] M. Karr. Summation in finite terms. J. ACM, 28:305-350, 1981.

[7] M. Karr. Theory of summation in finite terms. J. Symbolic Comput., 1:303-315, 1985.

[8] P. Paule and C. Schneider. Computer proofs of a new family of harmonic number identities. Adv. in Appl. Math., 31(2):359-378, 2003.

[9] C. Schneider. Symbolic summation in difference fields. Technical Report 01-17, RISC-Linz, J. Kepler University, November 2001. PhD Thesis.

[10] C. Schneider. A collection of denominator bounds to solve parameterized linear difference equations in $\Pi \Sigma$-fields. SFB-Report 02-20, J. Kepler University, Linz, November 2002. Submitted.

[11] C. Schneider. Degree bounds to find polynomial solutions of parameterized linear difference equations in $\Pi \Sigma$-fields. SFB-Report 02-21, J. Kepler University, Linz, November 2002. Submitted.

[12] C. Schneider. Solving parameterized linear difference equations in $\Pi \Sigma$-fields. SFB-Report 02-19, J. Kepler University, Linz, November 2002. Submitted.

[13] C. Schneider. Product representations in $\Pi \Sigma$-fields. Technical Report 02-24, RISC-Linz, J. Kepler University, 2003. Submitted.

[14] C. Schneider. Symbolic summation with single-nested sum extensions (extended version). SFB-Report 2004-7, J. Kepler University, Linz, 2004.

[15] D. Zeilberger. A fast algorithm for proving terminating hypergeometric identities. Discrete Math., 80(2):207-211, 1990. 